\title{
Corylus avellana L. Aroma Blueprint: Potent Odorants Signatures in the Volatilome of High Quality Hazelnuts
}

\author{
Simone Squara ${ }^{1}$, Federico Stilo ${ }^{1,2}$, Marta Cialiè Rosso ${ }^{1}$, Erica Liberto ${ }^{1}$, Nicola Spigolon ${ }^{3}$, \\ Giuseppe Genova ${ }^{3}$, Giuseppe Castello ${ }^{3}$, Carlo Bicchi ${ }^{1}$ and Chiara Cordero ${ }^{1 *}$ \\ ${ }^{1}$ Dipartimento di Scienza e Tecnologia del Farmaco, Università degli Studi di Torino, Turin, Italy, ${ }^{2}$ Laemmegroup - A Tentamus \\ Company, Turin, Italy, ${ }^{3}$ Soremartec Italia Srl, Cuneo, Italy
}

\section{OPEN ACCESS}

Edited by:

Valerio Cristofori,

University of Tuscia, Italy

Reviewed by:

Mario Di Guardo,

University of Catania, Italy Jasenka Gajdoš Kljusurić,

University of Zagreb, Croatia

*Correspondence:

Chiara Cordero

chiara.cordero@unito.it

Specialty section: This article was submitted to

Plant Breeding,

a section of the journal

Frontiers in Plant Science

Received: 20 December 2021

Accepted: 24 January 2022

Published: 03 March 2022

Citation:

Squara S, Stilo F, Cialiè Rosso M, Liberto E, Spigolon N, Genova G,

Castello G, Bicchi C and Cordero C

(2022) Corylus avellana L. Aroma Blueprint: Potent Odorants Signatures

in the Volatilome of High Quality

Hazelnuts.

Front. Plant Sci. 13:840028 doi: 10.3389/fpls.2022.840028
The volatilome of hazelnuts (Corylus avellana L.) encrypts information about phenotype expression as a function of cultivar/origin, post-harvest practices, and their impact on primary metabolome, storage conditions and shelf-life, spoilage, and quality deterioration. Moreover, within the bulk of detectable volatiles, just a few of them play a key role in defining distinctive aroma (i.e., aroma blueprint) and conferring characteristic hedonic profile. In particular, in raw hazelnuts, key-odorants as defined by sensomics are: 2,3-diethyl-5-methylpyrazine (musty and nutty); 2acetyl-1,4,5,6-tetrahydropyridine (caramel); 2-acetyl-1-pyrroline (popcorn-like); 2-acetyl3,4,5,6-tetrahydropyridine (roasted, carame); 3-(methylthio)-propanal (cooked potato); 3-(methylthio)propionaldehyde (musty, earthy); 3,7-dimethylocta-1,6-dien-3-ol/linalool (citrus, floral); 3-methyl-4-heptanone (fruity, nutty); and 5-methyl-(E)-2-hepten-4-one (nutty, fruity). Dry-roasting on hazelnut kernels triggers the formation of additional potent odorants, likely contributing to the pleasant aroma of roasted nuts. Whiting the newly formed aromas, 2,3-pentanedione (buttery); 2-propionyl-1-pyrroline (popcorn-like); 3-methylbutanal; (malty); 4-hydroxy-2,5-dimethyl-3(2H)-furanone (caramel); dimethyl trisulfide (sulfurous, cabbage) are worthy to be mentioned. The review focuses on high-quality hazelnuts adopted as premium primary material by the confectionery industry. Information on primary and secondary/specialized metabolites distribution introduces more specialized sections focused on volatilome chemical dimensions and their correlation to cultivar/origin, post-harvest practices and storage, and spoilage phenomena. Sensory-driven studies, based on sensomic principles, provide insights on the aroma blueprint of raw and roasted hazelnuts while robust correlations between non-volatile precursors and key-aroma compounds pose solid foundations to the conceptualization of aroma potential.

Keywords: Corylus avellana $L$., key-aroma compounds, metabolomics, chromatographic fingerprinting, hazelnuts volatilome, primary metabolites, volatile organic compounds (V.O.C.)

\section{INTRODUCTION}

European hazelnut (Corylus avellana L.) belongs to the Corylus genus, Betulaceae birch family, and is one of the 25 existing hazelnut species (Erdogan and Mehlenbacher, 2000); originally of the Black sea region, C. avellana L. has been cultivated since Roman times (Boccacci and Botta, 2009), but intensive production started to expand in the 1930s in the Langhe region in Piemonte (North-West 
of Italy) due to the demand from the confectionery industry, and since 1964 in Turkey (Bozoglu, 2005). C. avellana L. is the main species of interest for industrial applications due to high-quality characteristics such as larger kernels and thinner shells (Erdogan and Mehlenbacher, 2000).

Nowadays, hazelnuts represent a relatively small, yet consistent, market portion in constant growth both in developed countries and in emerging economies, which is projected to grow by over $10 \%$ in the next 5 years. According to the FAO (2019), during 2019 more than 1 million tons of in-shell hazelnuts were harvested. The global production during 2017-2019 is visually summarized in Figure 1. Turkey is the main producer since it covers more than $67 \%$ of the global production, followed by Italy $(\approx 12 \%)$, Azerbaijan $(\approx 5 \%)$, and the USA $(\approx 4 \%)$.

The Turkish production is mainly located in two areas along the Black Sea: the eastern area, which accounts for $60 \%$ and includes the provinces of Samsun, Ordu, Giresun, and Trabzon, and the western area, named Akçakoca, which accounts for the other $40 \%$ and includes the provinces of Sakarya, Zonguldak, Bolu, and Düzce. Turkish hazelnuts are usually supplied as a regional blend (e.g., Akçakoca blend and Giresun blend), and depending on the area of interest, different cultivars are more abundant: in the eastern area (Giresun/Ordu) the most prominent cultivars are the Tombul, Çakildak, Mincane, and Palaz, while in the western area (Akçakoca) the leading cultivars are the Karafindik, Mincane, Çakildak, and Foşa (ISLAM, 2018). Among these cultivars, Tombul is the most abundant and has been described as the best Turkish cultivar in terms of overall kernel quality (Balik et al., 2018)

Italy is the second-largest producer with four main production areas: Campania $(\approx 32 \%)$, where the cultivars are the Mortarella, San Giovanni, and Tonda di Giffoni; Piemonte $(\approx 30 \%)$, with the cultivar Tonda Gentile Trilobata; Lazio ( $\approx 25 \%)$, with the cultivars Tonda Gentile Romana and Nocchione; and Sicilia $(\approx 11 \%)$ where the cultivars are primarily used as fresh products and do not have an industrial interest.

In Azerbaijan, the main cultivar is the Ata-Baba which accounts for about $80 \%$ of the total hazelnut production and is concentrated in the Qabala and Qakh districts in the northwest of the country.

In the USA the production is concentrated in Oregon, on the pacific coast, which accounts for $\approx 99 \%$ of the total production. Barcelona is the main cultivar $(\approx 60 \%)$, but many cultivars from artificial breeding are continuously developed to improve the resistance to Phytocoptella avellanae, a mite affecting local trees. Table 1 reports a summary of the main cultivars and harvest regions of the four largest producers.

Table 2 summarizes the main characteristics of the most relevant hazelnut cultivars. It is important to highlight that industrially appealing hazelnut cultivar characteristics include thin shell, easy cuticle removal (high blanching rate), high shelling yield (kernel/nut ratio $>45 \%$ ), globular kernels, and a kernel caliber ideally around $13 \mathrm{~mm}$ (Caramiello et al., 2000).

This review focuses on the chemistry of the volatilome of high-quality hazelnuts; in particular, it systematically presents information about the distribution of potent odorants and keyaroma compounds in raw and roasted hazelnuts and critically discusses them given the increasing market demand for highquality products for the confectionery industry.

The impact of post-harvest practices, storage conditions, and roasting (i.e., the key-technological process) is examined for their impact on volatiles signatures and the development of positive and negative sensory attributes. The correlation between primary metabolites and key-odorants signatures, by the aroma potential concept (Cialiè Rosso et al., 2018, 2020, 2021), is also discussed as a new perspective for the application of modern omics strategies to hazelnut research (Miguel et al., 2011; Stilo et al., 2021a).

\section{HAZELNUTS COMPOSITION AND ITS CORRELATION TO SENSORY PROPERTIES}

A characteristic composition with a peculiar balance between primary and specialized (formerly referred to as secondary) metabolites is at the basis of the pleasant sensory profile and potential health benefits of hazelnuts. Taste-active components, interacting with chemoreceptors located in the oral cavity, trigger basic taste sensations (i.e., sweet, acid, bitter, salty, and umami); they are generally connoted by lower volatility accompanied by a high polarity and water solubility. In hazelnut, major taste active compounds are free amino acids, sugars, organic acids (i.e., primary metabolites), phenolic acids, and condensed tannins (i.e., specialized plant metabolites) (Alasalvar et al., 2010, 2012b).

Some of these non-volatile constituents belonging to the class of specialized metabolites (i.e., phenolic derivatives in aglycones or glycosides) can also trigger trigeminal sensations (i.e., chemestesis) while eliciting velvety and astringency sensations (Schieberle and Hofmann, 2011).

On the other hand, aroma-active components are characterized by low water solubility, medium-to-low polarity, and molecular weight below $300 \mathrm{Da}$. The interaction of odoractive volatiles with the array of Olfactory Receptors (ORs) in the olfactory epithelium activates a complex signals pattern, i.e., the Receptor Code (Firestein, 2001; Breer et al., 2006; Audouze et al., 2014; Dunkel et al., 2014). These olfactory stimuli activate the nervous system for cognitive mechanisms of learning and experience, and as the ultimate event the olfactory perception.

Aroma perception alone is responsible for up to $80 \%$ of the whole hedonic profile (Dunkel et al., 2014) of food. The synergy between taste and aroma perception, also referred to as flavor (Schieberle and Hofmann, 2011), is at the basis of positive consumer experience and of the hedonic quality of hazelnuts.

This review has hazelnut volatilome as a primary focus and, within the bulk of its detectable volatiles, those potent odorants capable of eliciting positive or negative sensations perceivable during hazelnut consumption.

The next section briefly summarizes the bulk composition of raw hazelnuts by presenting major primary and specialized metabolites classes with a focus on those components that are also potent odorant precursors.

\section{Primary Metabolites}

Hazelnuts are an all-round source of nutrients; in this perspective, the lipid fraction is the most abundant, it accounts 


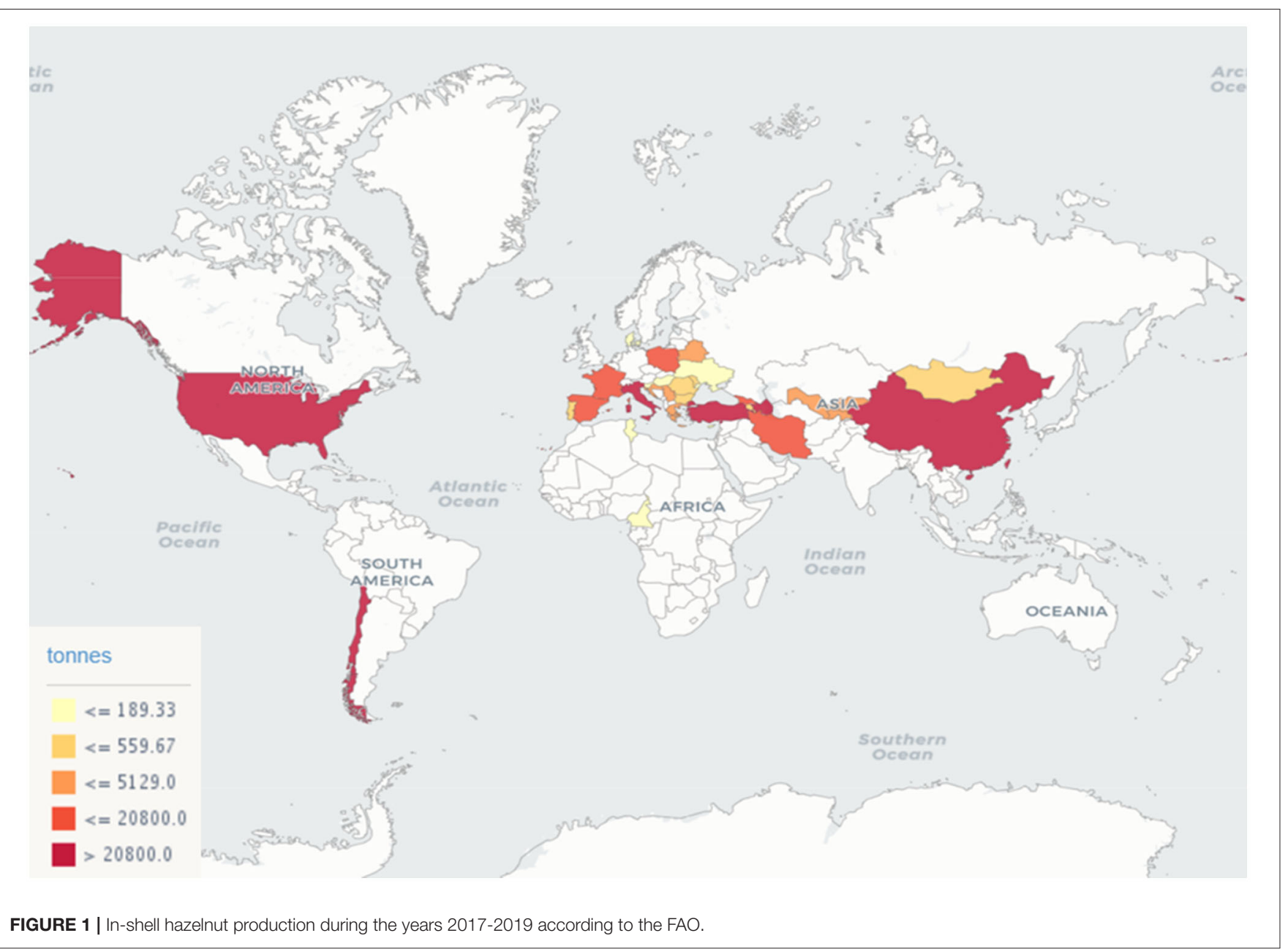

for $50-73 \%$ of the total composition (Köksal et al., 2006), followed by $10-22 \%$ of carbohydrates and $10-24 \%$ of proteins; moisture $(\approx 5 \%)$ and ashes $(\approx 3 \%)$ complete the profile.

\section{Lipids}

The lipid fraction is characterized by a saponifiable portion further classified in a polar fraction that accounts for $1.2 \%$ of the total amount and is represented by phosphatidylcoline, phosphatidylethanolamine, and phosphatidylinositol (Alasalvar et al., 2003b), and an apolar fraction of about 98.8\%, consisting of triacylglycerols of which the principal contributor is oleic acid

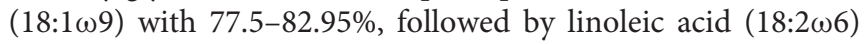
that is responsible for the $7.55-13.69 \%$ and by palmitic acid (16:0) that constitutes the $4.85-5.79 \%$ of the total. A summary of the most abundant fatty acids present in 16 varieties/cultivars are illustrated in Table 3 (Köksal et al., 2006).

The unsaponifiable fraction is mostly characterized by sterols, which differ in composition between cultivars and geographical origin, and decreases along with the shelf-life (Amaral et al., 2006; Ilyasoglu, 2015; Ghisoni et al., 2020). The three most abundant sterols are $\beta$-sitosterol, accounting for $85 \%$ of the total content, campesterol at $5-7 \%$, and $\Delta 5$-avenasterol, which achieves on average $2-4 \%$ of the total composition (Ilyasoglu, 2015). The sterol fraction plays an important role in authentication and frauds counteraction, it is used to identify olive oils adulterated with hazelnut oils of lower quality (Parcerisa et al., 1999; Zabaras and Gordon, 2004), as it might be further used in the discrimination of different hazelnut cultivars (Ghisoni et al., 2020).

The lipid fraction is at the basis of major sensory defects developed during hazelnut storage (Kinderlerer and Johnson, 1992; Alasalvar et al., 2003b; Azarbad and Jeleń, 2015; Ghirardello et al., 2016; Cialiè Rosso et al., 2018); unsaturated fatty acids are prone to autoxidation forming hydroperoxides derivatives further degraded in secondary products with lower polarity, molecular weight and odor threshold (OT) (Belitz et al., 2009). Moreover, TAGs are hydrolyzed and free fatty acids (FFAs) are more easily oxidized by the enzymatic activity, mainly promoted by endogenous esterases and lipases (Cialiè Rosso et al., 2021).

\section{Proteins and Free Amino Acids}

The hazelnut protein content is on average $18 \%$ in weight (Kamal-Eldin and Moreau, 2009). Proteins can be classified into two main groups: albumins and globulins with an aminoacidic 
TABLE 1 | Summary of the four main hazelnut producers with the regions of interest and dominant cultivars for each region.

\begin{tabular}{|c|c|c|c|}
\hline Country & Tons (\%) & $\begin{array}{l}\text { Regions and } \\
\text { Provinces }\end{array}$ & Cultivars \\
\hline \multirow[t]{2}{*}{ Turkey } & 655,348 (67\%) & $\begin{array}{l}\text { Giresun, Ordu, Samsun } \\
\text { and Trabzun - (60\%) }\end{array}$ & $\begin{array}{l}\text { Tombul, Çakildak, } \\
\text { Mincane and Palaz }\end{array}$ \\
\hline & & $\begin{array}{l}\text { Sakarya, Zonguldak, } \\
\text { Bolu and Düzce - (40\%) }\end{array}$ & $\begin{array}{l}\text { Karafindik, Mincane, } \\
\text { Çakildak and Foşa }\end{array}$ \\
\hline \multirow[t]{3}{*}{ Italy } & 120,837 (12\%) & Campania ( $\approx 32 \%)$ & $\begin{array}{l}\text { Mortarella, San Giovanni } \\
\text { and Tonda Giffoni }\end{array}$ \\
\hline & & Piemonte ( $\approx 30 \%)$ & Tonda Gentile Trilobata \\
\hline & & Lazio ( $\approx 25 \%)$ & $\begin{array}{l}\text { Tonda Gentile Romana, } \\
\text { Nocchione and Tonda } \\
\text { Giffoni }\end{array}$ \\
\hline Azerbaijan & $50,463(5 \%)$ & Qabala and Qakh & Ata-Baba \\
\hline USA & $38,406(4 \%)$ & Oregon & Barcelona \\
\hline
\end{tabular}

Percentage values refer to the global production during the years 2017-2019.

composition where glutamic acid (2.84-3.71 g/100 g), arginine $(1.87-2.21 \mathrm{~g} / 100 \mathrm{~g})$, and aspartic acid $(1.33-1.68 \mathrm{~g} / 100 \mathrm{~g})$ are the more abundant reaching $30 \%$ of the total fraction (Durak et al., 1999; Köksal et al., 2006; Ramalhosa et al., 2011). Table 4 shows in more detail the free amino acid profiles of hazelnuts from different geographical areas.

Caligiani et al. (2014) mapped the primary metabolome of selected raw and roasted hazelnuts from Tonda Gentile Trilobata (Piemonte TGT, Italy), Tonda Giffoni (Lazio, Italy), and Turkish varieties (Caligiani et al., 2014) by nuclear magnetic resonance (NMR) spectroscopy. Tryptophan was the discriminant marker for the Turkish hazelnuts while higher concentrations of choline and acetic acid characterized TGT samples.

Besides their role as markers for authentication, amino acids are also non-volatile precursors of several potent odorants responsible for pleasant notes in roasted hazelnuts. In a recent study, Cialiè Rosso et al. $(2020,2021)$ investigated the amino acidic patterns in raw and roasted hazelnuts while observing linear correlations between aroma precursors in raw hazelnuts and potent odorants in lab-scale roasted hazelnuts.

\section{Carbohydrates}

Carbohydrates can reach $26 \%$ of the total composition; they can be further divided into dietary fiber, sugars, and starch (Coelho et al., 2007; Alasalvar and Shahidi, 2008). In unroasted hazelnuts, the percentage of total non-resistant and resistant starch expressed as \% on a dry basis (d.b.), is $2.71 \pm 0.08,1.190 \pm$ 0.07 , and $1.52 \pm 0.01$, respectively (Alasalvar and Shahidi, 2008).

Sugars reach $17 \%$ of the total composition; they include di-saccharides (sucrose, stachyose, raffinose), monosaccharides (glucose and fructose), and polyalcohols (myo-inositol). The total sugar content of hazelnut is, on average, around $3.58 \mathrm{~g} / 100 \mathrm{~g}$, and the sucrose achieves about $74 \%$ of the total (Alasalvar and Shahidi, 2008; Sciubba et al., 2014). Sugars contribute directly and indirectly to hazelnut sensorial profile: they are responsible for the sweet taste of raw nuts and represent fundamental precursors of aroma active compounds since they react within the Maillard reaction framework and degrade during roasting (Cristofori et al., 2008; Kiefl, 2013; Kiefl and Schieberle, 2013; Taş and Gökmen, 2018).

Bonvehí and Coll (1993) studied the carbohydrates fraction and its variations as a function of varieties/cultivar and harvest region; their findings indicated that starch and fiber were almost stable while soluble sugars were highly variable within varieties, with the mountain harvested varieties containing a higher sucrose amount.

\section{Specialized Metabolites}

Phenolic derivatives are the most abundant compounds in hazelnut kernels amongst specialized metabolites (Shahidi et al., 2007; Alasalvar and Shahidi, 2008; Alasalvar and Bolling, 2015; Bottone et al., 2019). Among them, phenolic acids of the hydroxybenzoic acid series are represented by gallic acid, the most abundant, followed by $p$-hydroxybenzoic acid, salicylic acid, 4-hydroxysalicylic acid, vanillic acid, and syringic acid. Among the hydroxycinnamic derivatives, $o$ - and $p$-coumaric acid, caffeic acid, ferulic and isoferulic acid, and sinapic acid were identified in hazelnut kernels (Yuan et al., 2018).

Polyphenols are instead represented by quercetin, myricetin, and rutin, present in kernels as aglycones and/or as $O$ glycosides (e.g., quercetin 3-rhamnoside, quercetin-3-glucoside, and myricetin 3-rhamnoside) (Bottone et al., 2019). Among flavonoids, catechin, epicatechin, and epigallocatechin were identified in kernels (Prosperini et al., 2009; Fanali et al., 2018) accompanied by a complex fraction of their polymers (i.e., proanthocyanidins and condensed tannins) with procyanidins A2, B1, and B2 as major congeners (Fanali et al., 2018; Bottone et al., 2019).

Phenol signatures were effectively exploited for cultivar discrimination by Ciarmiello et al. (2014) who analyzed 29 European cultivars and, based on the total polyphenolic content and the qualitative composition, defined some potential markers for quality control.

Recently, by untargeted metabolomic investigation based on liquid chromatography and high-resolution mass spectrometry (LC-ESI-qTOF MS), Ghisoni et al. (2020) profiled six hazelnut cultivars harvested in Chile, Georgia, Italy, and Turkey. More than 1,000 polyphenols and sterols were annotated and tracked among samples. Flavonoids (anthocyanins, flavanols, and flavonols), phenolic acids (mainly hydroxycinnamics) together with sterols (i.e., cholesterol, ergosterol, and stigmasterol derivatives) were defined as putative markers for geographical origin discrimination.

Phenols and polyphenols, also present in hazelnut perisperm cuticle, are responsible for the taste and chemesthetic attributes (bitterness, astringency, velvety sensations), and during roasting may form phenolic volatiles eliciting smoky and phenolic odors, the latter being distinctive of high-quality cultivars (BurdackFreitag and Schieberle, 2012; Kiefl et al., 2013).

Another class of specialized metabolites is that of monoterpenoids: they are biosynthesized from C5 precursors (i.e., dimethyl allyl pyrophosphate and isopentenyl pyrophosphate) in plants and are present in raw and roasted hazelnut kernels as distinctive native signatures. The most 
TABLE 2 | List of the most industrially used cultivars and their characteristics.

\begin{tabular}{|c|c|c|}
\hline Cultivars & Area of cultivation & Characteristics \\
\hline Ata-Baba & Azerbaijan & Small nut with plane-round shape, thin shell \\
\hline Barcelona & Oregon (USA), Chile, France & Large nut with conical shape $(\mathrm{RI}=0.92), 43 \%$ kernel/nut ratio, intermediate blanching rate, thick shell. \\
\hline Çakildak & Ordu (TR) & Small nut with globular shape $(\mathrm{RI}=1), 46 \% \mathrm{kernel} /$ nut ratio, high blanching rate, thin shell. \\
\hline Foşa & Trabzon (TR), Akçakoca (TR) & Small nut with globular shape $(\mathrm{Rl}=0.97), 50 \%$ kernel/nut ratio, high blanching rate, thin shell. Good flavor. \\
\hline Karafindik & Akçakoca (TR) & Small nut with a long shape $(\mathrm{RI}=0.83), 33.9 \% \mathrm{kernel} /$ nut ratio, high blanching rate, thin shell \\
\hline Mincane & Akçakoca (TR) & Small nut with a long shape $(\mathrm{RI}=0.87), 47 \% \mathrm{kernel} /$ nut ratio, high blanching rate, thin shell \\
\hline Mortarella & Campania (IT) & $\begin{array}{l}\text { Medium to small nut with short cylindrical shape }(\mathrm{RI}=0.78), 45 \% \mathrm{kernel} / \text { nut ratio, high blanching rate, thin } \\
\text { shell. Excellent flavor. }\end{array}$ \\
\hline Nocchione & Lazio (IT) & $\begin{array}{l}\text { Medium to large nut with globular shape }(\mathrm{RI}=0.97) \text {, 38\% kernel/nut ratio, intermediate blanching rate, thick } \\
\text { shell. }\end{array}$ \\
\hline Palaz & Ordu (TR) & $\begin{array}{l}\text { Medium nut with globular shape }(\mathrm{Rl}=0.99), 47 \% \mathrm{kernel} / \text { nut ratio, very high blanching rate, thin shell. Good } \\
\text { flavor. }\end{array}$ \\
\hline San giovanni & Lazio (IT) & $\begin{array}{l}\text { Medium nut with short cylindrical shape }(\mathrm{RI}=0.76), 46 \% \text { kernel/nut ratio, intermediate blanching rate, } \\
\text { intermediate shell thickness. }\end{array}$ \\
\hline Tombul & Turkey (All regions) & $\begin{array}{l}\text { Small nut with conical shape }(\mathrm{RI}=0.83), 50 \% \mathrm{kernel} / \text { nut ratio, very high blanching rate, thin shell. Excellent } \\
\text { flavor. }\end{array}$ \\
\hline Tonda giffoni & Campania (IT) & $\begin{array}{l}\text { Medium nut with globular shape }(\mathrm{RI}=1), 46 \% \text { kernel/nut ratio, high blanching rate, thin shell. Excellent flavor. } \\
\text { European labeling: PDO-IT-0573-AM01 }\end{array}$ \\
\hline Tonda gentile trilobata & Piemonte (IT) & $\begin{array}{l}\text { Small to medium nut with flat shape }(\mathrm{RI}=0.98), 45.5 \% \text { kernel/nut ratio, high blanching rate, thin shell. } \\
\text { Excellent flavor. European labeling: PGI-IT-0305-AM03 }\end{array}$ \\
\hline Tonda gentile romana & Lazio (IT) & $\begin{array}{l}\text { Medium nut with globular shape }(\mathrm{RI}=0.98), 45 \% \mathrm{kernel} / \mathrm{nut} \text { ratio, intermediate blanching rate, thin shell. } \\
\text { Very good aroma. European labeling: PDO-IT-0573-AM01 }\end{array}$ \\
\hline
\end{tabular}

TABLE 3 | Fatty acid composition of 16 hazelnut varieties, data expressed in $\mathrm{g} / 100 \mathrm{~g}$.

\begin{tabular}{lcccccc}
\hline Variety & C16:0 & C16:1 & C18:0 & C18:1 & C18:2 & C18:3 \\
\hline Cavacava & 5.87 & 0.22 & 2.37 & 78.8 & 12.7 & 0.069 \\
Çakildak & 4.89 & 0.32 & 2.15 & 80.7 & 11.9 & 0.059 \\
Foşa & 5.62 & 0.37 & 1.70 & 79.0 & 13.2 & 0.074 \\
Incekara & 5.67 & 0.32 & 1.76 & 79.5 & 12.7 & 0.073 \\
Kalinkara & 5.71 & 0.42 & 2.42 & 79.5 & 11.9 & 0.067 \\
Kan & 5.72 & 0.32 & 2.30 & 81.8 & 9.82 & 0.053 \\
Karafindik & 5.62 & 0.28 & 2.37 & 78.9 & 12.8 & 0.058 \\
Kargalak & 4.89 & 0.42 & 0.86 & 81.0 & 12.7 & 0.067 \\
Kuş & 5.69 & $<$ LOQ & 0.87 & 79.9 & 13.5 & 0.076 \\
Mincane & 5.02 & 0.38 & 1.90 & 82.8 & 9.89 & 0.029 \\
Palaz & 4.87 & 0.34 & 2.13 & 77.6 & 15.0 & 0.076 \\
Sivri & 4.72 & 0.42 & 2.49 & 79.2 & 13.2 & $<$ LOQ \\
Tombul & 5.17 & 0.48 & 1.75 & 77.8 & 14.8 & 0.054 \\
Uzunmusa & 5.70 & 0.46 & 1.41 & 78.8 & 13.6 & 0.069 \\
Yassi Badem & 4.87 & 0.28 & 1.43 & 81.1 & 12.2 & 0.046 \\
Yuvarlak Badem & 5.66 & 0.36 & 0.87 & 74.2 & 18.73 & $<$ LOQ \\
\hline TableaptedbyKô & & & & & &
\end{tabular}

Table adapted by Köksal et al. (2006).

reported are pinenes ( $\alpha$-pinene and $\beta$-pinene) (Alasalvar et al., 2003a; Cordero et al., 2010; Burdack-Freitag and Schieberle, 2012; Kiefl, 2013; Cialiè Rosso et al., 2018), linalool and limonene (Burdack-Freitag and Schieberle, 2012; Cialiè Rosso et al., 2018), $\delta$-3-carene (Alasalvar et al., 2003a), and $\beta$-caryophyllene, a sesquiterpenoid detected in Turkish Tombul hazelnuts (Alasalvar et al., 2003a).

Recently, by applying comprehensive two-dimensional gas chromatography (GC×GC), which enables highly effective fingerprinting of volatiles in many foods, a detailed signature of terpenoids was delineated. It includes $\alpha$-pinene, $\beta$-pinene, $(E)$-p2 -menthen-1-ol, camphene, $\delta$-3-carene, $\alpha$-thujene, $\gamma$-terpinene, sabinene, limonene, cis-sabinene hydrate, $\alpha$-terpinolene, $\beta$ phellandrene, and p-cymene (Kiefl, 2013; Kiefl et al., 2013; Nicolotti et al., 2013b; Cialiè Rosso et al., 2018). In particular with specialized metabolites, $\alpha$-damascone and (E)- $\beta$-damascenone, nor-isoprenoids formed by oxidative cleavage of carotenoids, are of relevance for hazelnut aroma (Burdack-Freitag and Schieberle, 2010).

\section{HAZELNUT VOLATILOME}

The volatilome, also referred to as volatome (Phillips et al., 2013; Broza et al., 2015), "contains all of the volatile metabolites as well as other volatile organic and inorganic compounds that originate from an organism" (Amann et al., 2014), super-organism, or ecosystem. In line with this definition, all volatile metabolites present in the volatilome belongs to the sample's metabolome, although in this complex fraction it could be present degradation components or exogenously formed compounds not generated by plant metabolic processes [e.g., environmental contaminants, compounds formed by bacteria and molds metabolic processesmicrobial cloud (Meadow et al., 2015), etc.].

The volatilome is therefore a distinct entity from the metabolome, it gives access to a higher level of information 
TABLE 4 | Amino acids composition ( $\mathrm{g} / 100 \mathrm{~g})$ of hazelnuts from different geographical origin.

\begin{tabular}{lcccc}
\hline Amino acids & Tombul & Turkey $^{\mathrm{a}}$ & USA $^{\mathrm{b}}$ & New Zealand $^{\mathrm{c}}$ \\
\hline Alanine & 0.7 & 0.72 & 0.73 & 0.54 \\
Arginine & 2.16 & 2 & 2.21 & 1.87 \\
Aspartic acid & 1.52 & 1.49 & 1.68 & 1.33 \\
Cysteine & 0.46 & Unidentifiable & 0.28 & 0.27 \\
Glutamic acid & 3.13 & 2.84 & 3.71 & 2.86 \\
Glycine & 0.71 & 0.64 & 0.72 & 0.54 \\
Histidine & 0.45 & 0.42 & 0.43 & 0.32 \\
Hydroxyproline & 0.06 & Unidentifiable & Unidentifiable & Unidentifiable \\
Isoleucine & 0.58 & 0.56 & 0.55 & 0.47 \\
Leucine & 1.07 & 1.15 & 1.06 & 0.82 \\
Lysine & 0.41 & 0.45 & 0.42 & 0.42 \\
Methionine & 0.23 & 0.16 & 0.22 & 0.18 \\
Phenylalanine & 0.66 & 0.64 & 0.66 & 0.56 \\
Proline & 0.56 & 0.59 & 0.56 & 0.49 \\
Serine & 0.65 & 0.72 & 0.74 & 0.56 \\
Threonine & 0.53 & 0.46 & 0.5 & 0.39 \\
Tryptophan & 0.04 & Unidentifiable & 0.19 & Unidentifiable \\
Tyrosine & 0.53 & 0.47 & 0.36 & 0.4 \\
Valine & 0.71 & 0.66 & 0.7 & 0.6 \\
\hline Tabe & & & & 0.56 \\
\hline
\end{tabular}

Table adapted from Alasalvar et al. (2003b) and Alasalvar and Shahidi (2008). "a" varieties Aci, Cavcava, Çakildak, Foşa, Incekara, Kalinkara, Kan, Karafindik, Kargalak, Kus, Mincane, Palaz, Sivri, Tombul, Uzunmusa, Yassi Badem, and Yuvarlak Badem. "b" unknown varieties. "c": varieties Whiteheart, Barcelona, Butler, Ennis, Tonda di Giffoni, and Campanica.

about many biological phenomena related to plant and food quality through its multiple chemical dimensions (Giddings, 1995). The ultimate analytical solutions to investigate food volatilome are those adopting high-resolution separations (e.g., mono-dimensional gas chromatography-1DGC; heartcut two-dimensional gas chromatography -H/C-2DGC; or comprehensive two-dimensional gas chromatography $\mathrm{GC} \times \mathrm{GC}$ ) combined to low-resolution or high-resolution mass spectrometry (MS). Insights on analytical strategies for comprehensive investigations of food volatiles are outside the scope of this review; however, for interested readers, here follow some reference papers of interest (Sides et al., 2000; Tranchida et al., 2013; Cordero et al., 2015, 2019; Franchina et al., 2016; Pedrotti et al., 2021; Stilo et al., 2021a).

The food volatilome is a complex mixture of volatiles belonging to several different chemical classes as a function of the main metabolisms and reactions contributing to its expression. Regarding hazelnuts, native volatiles are those formed along with the terpenoid biosynthesis, from isopentenyl pyrophosphate and dimethyl allyl pyrophosphate as precursors (Dewick, 1986). They are a direct expression of the plant phenotype although recent findings correlated their presence to bacteria and mold development during storage (Stilo et al., 2021b).

Other important volatiles present in the hazelnut volatilome are secondary products of lipid oxidation. They are degradation products ( $\beta$-scission and hydroperoxide epi-dioxide decomposition) of fatty acids hydroperoxides formed by lipids autoxidation. Within this group, several low molecular weight carbonyl derivatives (linear saturated aldehydes, unsaturated aldehydes, methyl-ketones), hydrocarbons, alcohols, and shortchain fatty acids can be found (Kinderlerer and Johnson, 1992; Belitz et al., 2009). When post-harvest practices do not properly stabilize kernels before storage, fruit germination might occur as well as bacteria and molds could find optimal conditions to grow (water activity $-a_{w}$, temperature, and substrates availability) increasing volatilome chemical complexity. As a consequence, primary and secondary alcohols, carboxylic acids from fermentation reactions (Cialiè Rosso et al., 2018), lactones by cyclization of hydroxyl substituted fatty acids, furans by glucose and reducing sugars degradation, and some aromatic derivatives (benzaldehyde, phenyl ethyl alcohol, phenyl ethyl acetaldehyde, alkylated phenol derivatives) by non-volatile precursors like amino acids and phenolic compounds can be found (Cialiè Rosso et al., 2018).

Industrial processing has its impact on primary food materials and is generally designed to obtain optimal hedonic properties including desirable flavor, texture, and color (Alasalvar et al., 2003a, 2006; Nicolotti et al., 2013a). Specifically, for hazelnuts, industrial roasting promotes the formation of a complex array of volatile compounds that concur with the volatilome complexity. Medium-polarity, low-molecular-weight technological markers belong to many different classes: alcohols, Strecker aldehydes, ketones, and di-carbonyls formed within the Maillard reaction framework (Cordero et al., 2008, 2010; Kiefl et al., 2013), acids, esters, lactones, sulfur derivatives, and alkylated heterocycles (furans, pyrazines, pyrroles, thiophenes, aromatic compounds, phenols, pyridines, thiazoles, oxazoles) (Saklar et al., 2001; Alasalvar et al., 2003a; Seyhan et al., 2007; Burdack-Freitag and Schieberle, 2010, 2012; Kiefl et al., 2012, 2013; Kiefl, 2013).

The next paragraphs report and discuss major findings of the hazelnut volatilome by focusing on specific functional variables with a strong correlation to sensory quality.

\section{Cultivar/Origin Signatures in the Hazelnut Volatilome}

Alasalvar et al. (2004, 2012a) dedicated several research projects to delineate distinctive signatures of potent odorants in highquality hazelnuts from Turkey. In a study on Giresun and Tombul hazelnuts (harvest year 2001), authors applied dynamic headspace analysis (DHA) combined with gas chromatography coupled with mass spectrometry (GC-MS) for high informative profiling of volatiles. A total of 79 volatile compounds were identified by matching linear retention index $\left(I^{T}\right)$ and EIMS spectral signatures with authentic standards and/or data from commercial databases. A total of 39 compounds were detected in raw hazelnuts; of them, 32 were identified as ketones (10), aldehydes (8), alcohols (5), aromatic hydrocarbons (5), and furans (4). Some of these components, as potent odorants, were positively correlated with specific and peculiar odor qualities revealed by descriptive sensory analysis (DSA) run by a trained panel. 
Of these compounds, within ketones, the most odor impacting was 5-methyl-(E)-2-hepten-4-one (i.e., filbertone) with a typical nutty and hazelnut-like aroma. (E)-3-penten-2-one was correlated to a fruity odor while 2,3-pentanedione to sweet, buttery, and caramel-like notes. In the aldehydes class, eight congeners were found in raw kernels, 2-methylpropanal, 2- and 3-methylbutanal were reported to be responsible for fruity, malty, nutty, and chocolate-like odors. In addition, linear saturated and monounsaturated aldehydes (i.e., hexanal, heptanal, nonanal, (E)-2-hexanal, and (E,E)-2,4-hexadienal) were responsible for perceivable green, fatty, sweet floral, and fruity notes. They are strongly correlated to lipid autoxidation and have a role in the sensorial quality degradation of kernels along with shelf-life (Cialiè Rosso et al., 2018).

Five alcohols were detected by dynamic headspace (DHS-)GC-MS in raw hazelnuts: 3-methyl-1-butanol was correlated to dark chocolate, pungent, and sweet odors while 1-pentanol was correlated with rancid, burnt, winelike notes, 1-hexanol typical of green and fatty smells, 1-octanol and 1-octen-3-ol, most probably formed by fatty acids hydroperoxide decomposition, were connoted by musty and mushroom-like odors.

Aromatic hydrocarbons, like toluene, 1,2,4-trimethylbenzene, and 1,2,3-trimethylbenzene were more abundant in roasted kernels although their presence was above the method limit of detection (LOD) for raw hazelnuts.

Raw hazelnut volatilome mapping greatly improved, in terms of the number of volatiles detected and the method's sensitivity, with the application of GC $\times$ GC-TOF MS combined with high concentration capacity (HCC) sampling (Bicchi et al., 2004). Cialiè Rosso et al. reported the reliable identification of 133 volatiles in raw hazelnuts from Ordu (Turkey), and Lazio (Tonda Gentile Romana, Italy); most of them were already cross-mapped in other cultivars/origins (e.g., Tonda Giffoni, Tonda Gentile Trilobata, Mortarella, Akçakoca, Giresun, Trabzon, and Chile) by GC $\times$ GC-qMS (Cordero et al., 2010; Nicolotti et al., 2013a). Table 5 reports a consensus list of characteristic volatiles together with their experimental $I^{T}$ on polar columns, odor qualities, and OTs in oil or air.

\section{Effect of Post-harvest and Storage on Volatilome Signatures}

The evolution of raw hazelnut volatilome along shelf-life was explored by Cialiè Rosso et al. (2018) in a study on commercial samples of Tonda Gentile Romana and on Ordu hazelnuts harvested in 2014. Samples were subjected to traditional sundrying (D1 - $\approx 30 / 35^{\circ} \mathrm{C}$ ) or artificial drying (D2) at low temperatures $\left(\approx 18 / 20^{\circ} \mathrm{C}\right)$ in industrial plants. To study the effect of storage conditions, $5^{\circ}$ and $18^{\circ} \mathrm{C} \pm 0.1$ were tested in combination with atmosphere composition as regular (NA: 78\% $\mathrm{N}_{2}-21 \% \mathrm{O}_{2}$ ) or modified (MA $99 \% \mathrm{~N}_{2}-1 \% \mathrm{O}_{2}$ ).

Volatiles and potent odorants were sampled by headspace solid-phase microextraction (HS-SPME) and analyzed by GC $\times$ GC-TOF MS equipped with a thermal modulator. Analytical conditions enabled a suitable sensitivity by including in the fingerprinting/profiling process potent odorants and several key-aroma compounds (Burdack-Freitag and Schieberle, 2012; Kiefl et al., 2013).

The pattern of 133 known analytes, identified by matching EIMS fragmentation patterns with those collected in commercial and in-house databases and $I^{T}$ calculated on the ${ }^{1} \mathrm{D}( \pm 15$ units of tolerance), was explored by multivariate statistics to highlight relevant features (i.e., components) with meaningful variations along storage time and as a function of storage conditions.

Explorative Principal Component Analysis (PCA) on analytes' response data indicated a natural conformation of sample groups according to cultivar/geographical origin, followed by the impact of a secondary variable, post-harvest drying conditions. Supervised univariate analysis by Fisher ratio (F), highlighted as relevant variables for post-harvest a series of linear and branched alcohols (2-heptanol, 2-methyl-1-propanol, 3-methyl-1-butanol, 2-ethyl-1-hexanol, benzyl alcohol), several esters (ethyl acetate, butyl butanoate, 2-methyl-butyl propanoate), and acetic acid. Most of them were already associated with nut ripening or fermentation (Zhou et al., 2013). Of interest, 3-methyl-1-butanol (i.e., isoamyl alcohol), a well-known fermentation product in must and wines, is formed from L-leucine. 2-methyl-1-propanol has instead L-valine as a precursor, while 2-heptanol is formed in tomatoes during ripening by $\beta$-ketoacids hydrolysis and subsequent decarboxylation (Fridman, 2005), and 2-ethyl-1hexanol has been found in fermented soybean (Han et al., 2001).

By observing the evolution of 37 potent odorants within the detectable volatilome, Cialiè Rosso et al. (2018) confirmed the dominant role of drying conditions above cultivar/origin. Moreover, most potent odorants, with OTs up to $2,500 \mu \mathrm{g} / \mathrm{L}$, were closely correlated $(r>0.800)$ to storage time. Of them, 1-heptanol (green, chemical), 2-octanol (metal, burnt), 1-octen3-ol (mushroom), (E)-2-heptenal (fatty, almond), hexanal (leaflike, green), heptanal (fatty), octanal (fatty), and nonanal (tallowy, fruity) are of great interest, since they might impart unpleasant notes in hazelnuts stored within 12 months. Some of the selected potent odorants showed increasing trends over time, achieving their maximum abundance at 12 months of storage. Those correlated to lipid oxidation, i.e., degradation products of fatty acids hydroperoxides (i.e., hexanal, octanal and (E)-2heptanal) eliciting fatty and green-leafy notes (Pastorelli et al., 2007; Ghirardello et al., 2013), had a marked increase over time with higher relative ratios in samples subjected to sun drying. For those samples dried at lower temperatures in industrial plants, i.e., Tonda Gentile Romana, limited oxidation was detected, with amounts of hexanal and octanal at 2.6 and 2.8 times lower compared to standard drying conditions.

Moreover, 2-octanol and 1-octen-3-ol, formed by linoleic acid hydroperoxides cleavage promoted by fungal lipoxygenase/hydroperoxide lyase enzymes (Hung et al., 2014), are likely responsible for the metallic and mushroom-like notes. Their relative abundance was higher in Ordu samples accompanied by a marked increase with storage conducted in less protective conditions $\left(18^{\circ} \mathrm{C}\right.$ and $\left.\mathrm{NA} 78 \% \mathrm{~N}_{2}-21 \% \mathrm{O}_{2}\right)$. On the other hand, the same analytes were below method LOD in Tonda Gentile Romana hazelnuts dried at low temperatures.

Results on oxidative stability/instability are likely correlated to hazelnuts fatty acids profiles reported in several studies 
TABLE 5 | Volatilome composition detected in raw and roasted hazelnut samples.

\begin{tabular}{|c|c|c|c|c|c|c|}
\hline Compound & CAS & Odor quality & OT $(\mu \mathrm{g} / \mathrm{kg})$ & ${ }^{1} \mathrm{D} I^{T}$ (polar) & Chemical class & Reference \\
\hline (E)-2-Decenal & $3913-71-1$ & Fatty, tallowy, orange-like & $101^{\wedge}$ & 1,629 & Aldehydes & $A, B$ \\
\hline (E)-2-Heptenal & $18829-55-5$ & Fatty, almond-like & $14^{\times}$ & 1,306 & Aldehydes & $A, B$ \\
\hline (E)-2-Hexenal & $6728-26-3$ & Green, fresh & $0.42^{x}$ & 1,202 & Aldehydes & $A, C$ \\
\hline (E)-2-Nonenal & $18829-56-6$ & Fatty, cucumber-like & $4.1^{\wedge}$ & 1,510 & Aldehydes & $A, B$ \\
\hline (E)-2-Octenal & $2548-87-0$ & Fatty, nutty & $50^{\wedge}$ & 1,415 & Aldehydes & $A, B$ \\
\hline (E)-3-Hepten-2-one & $1119-44-4$ & 1 & 1 & 1,277 & Ketones & $A$ \\
\hline (E)-3-Penten-2-one & 3102-33-8 & $\backslash$ & 1 & 1,102 & Ketones & $B, C, D$ \\
\hline (E)-Methyl-2-octenoate & 7367-81-9 & Sweet, fruity & 1 & 1,386 & Esters & A \\
\hline$(\mathrm{E}, \mathrm{E})-2,4$-Decadienal & $25152-84-5$ & Fatty & $166^{*}$ & 1,783 & Aldehydes & $E$ \\
\hline (E,E)-2,4-Hexadienal & $142-83-6$ & Green & $270^{x}$ & 1,411 & Aldehydes & C \\
\hline 1,2,3-Trimethylbenzene & $526-73-8$ & 1 & 1 & 1,301 & Aromatics & $A, C$ \\
\hline 1,2,4-Trimethylbenzene & $95-63-6$ & 1 & 1 & 1,283 & Aromatics & C \\
\hline 1,3-Dimethylbenzene & $108-38-3$ & Plastic & $1,000 \times £$ & 1,122 & Aromatics & $A$ \\
\hline 1-Butanol & $71-36-3$ & Fruity & $3,112^{x}$ & 1,089 & Alcohols & B \\
\hline 1-Cyclopentyl ethanone & $6004-60-0$ & 1 & $\backslash$ & 1,203 & Ketones & C \\
\hline 1-Decene & $872-05-9$ & 1 & $\backslash$ & 1,033 & Alkanes & $A$ \\
\hline 1-Dodecanol & $112-53-8$ & Waxy, soapy & $1,200 \times £$ & 1,960 & Alcohols & $A$ \\
\hline 1-Heptanol & $111-70-6$ & Cucumber, citrus-like & $20,000^{x}$ & 1,438 & Alcohols & $A, B, C$ \\
\hline 1-Hepten-3-ol & $4938-52-7$ & Green, oily, tomato-like & $3000^{x}$ & 1,367 & Alcohols & B \\
\hline 1-Heptene & $592-76-7$ & 1 & $\backslash$ & 828 & Alkanes & $A$ \\
\hline 1-Hexanol & $111-27-3$ & Green, flowery & $400^{\times}$ & 1,338 & Alcohols & $A, B, C$ \\
\hline 1-Octene & $111-66-0$ & Gasoline & $2,000^{x}$ & 851 & Alkanes & $A$ \\
\hline 1-Pentanol & $71-41-0$ & Balsamic & $470^{\times}$ & 1,239 & Alcohols & $A, B, C$ \\
\hline 1-Tetradecanol & $112-72-1$ & Waxy, fruity & $5,000 \times \$$ & 2,183 & Alcohols & A \\
\hline 2-(1-Pyrrolyl)ethanol & $22186-60-3$ & 1 & 1 & 1,949 & Alcohols & B \\
\hline 2-(2-Ethoxyethoxy)-ethanol & $111-90-0$ & Slightly ethereal & $6,000 \times £$ & 1,572 & Alcohols & A \\
\hline 2(3H)-Furanone & $20825-71-2$ & 1 & 1 & 1,760 & Lactones & $\mathrm{B}$ \\
\hline 2,2-Dimethyl-3-hexanone & $5405-79-8$ & 1 & 1 & 1,070 & Ketones & $\mathrm{B}$ \\
\hline 2,3,5-Trimethylfuran & $10504-04-8$ & 1 & 1 & 1,037 & Heterocycles & $B, C, D$ \\
\hline 2,3,5-Trimethylnaphthalene & $2245-38-7$ & Earthy & $\backslash$ & 1,562 & Aromatics & $\mathrm{C}$ \\
\hline 2,3,5-Trimethylpyrazine & $14667-55-1$ & Earthy & $290^{x}$ & 1,376 & Pyrazines & $E, C, B, D$ \\
\hline 2,3-Butanediol & $513-85-9$ & Fruity, onion-like & $20,000^{\times}$ & 1,593 & Alcohols & D \\
\hline 2,3-Butanedione & 431-03-8 & Buttery & $9.2^{\wedge}$ & 983 & Ketones & $E, B$ \\
\hline 2,3-Diethyl-5-methylpyrazine & $18138-04-0$ & Earthy, roasty & $0.5^{\star}$ & 1,470 & Pyrazines & $\mathrm{E}$ \\
\hline 2,3-Dimethylpyrazine & $5910-89-4$ & Nutty, cocoa-like & $880 \times £$ & 1,338 & Pyrazines & $\mathrm{D}$ \\
\hline 2,3-Pentanedione & $600-14-6$ & Buttery & $0.3^{\wedge}$ & 1,049 & Ketones & E, C, B, D \\
\hline 2,4-Dimethyl-3-pentanol & $600-36-2$ & 1 & 1 & 1,409 & Alcohols & $A, B$ \\
\hline 2,5-Dimethylfuran & $625-86-5$ & Ethereal, chemical & $100,000 \times £$ & 940 & Aldehydes & $A, B, C, D$ \\
\hline 2,5-Dimethylpyrazine & $123-32-0$ & Nutty, cocoa-like & $2,600^{*}$ & 1,317 & Pyrazines & $C, D$ \\
\hline
\end{tabular}


TABLE 5 | Continued

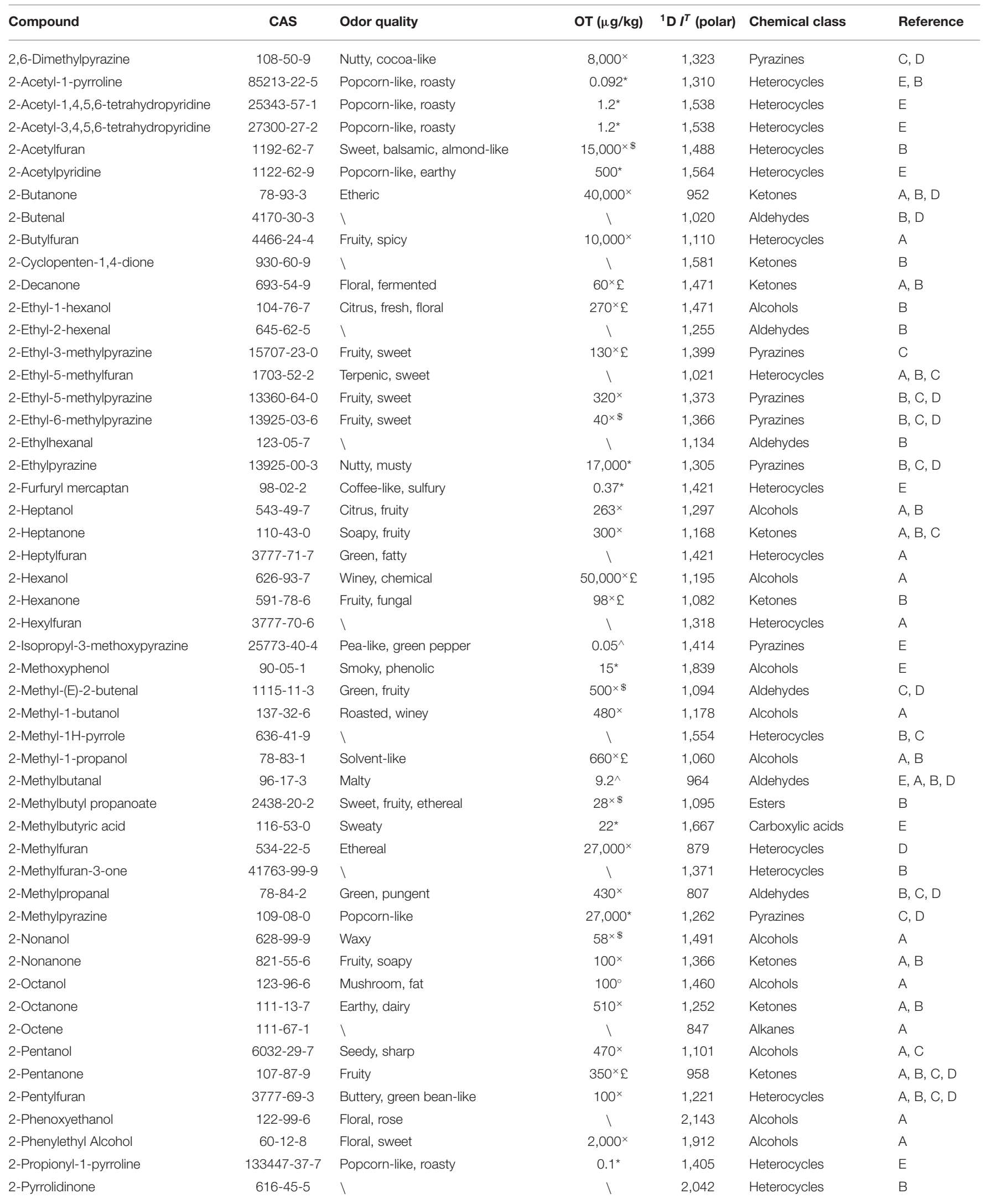


TABLE 5 | Continued

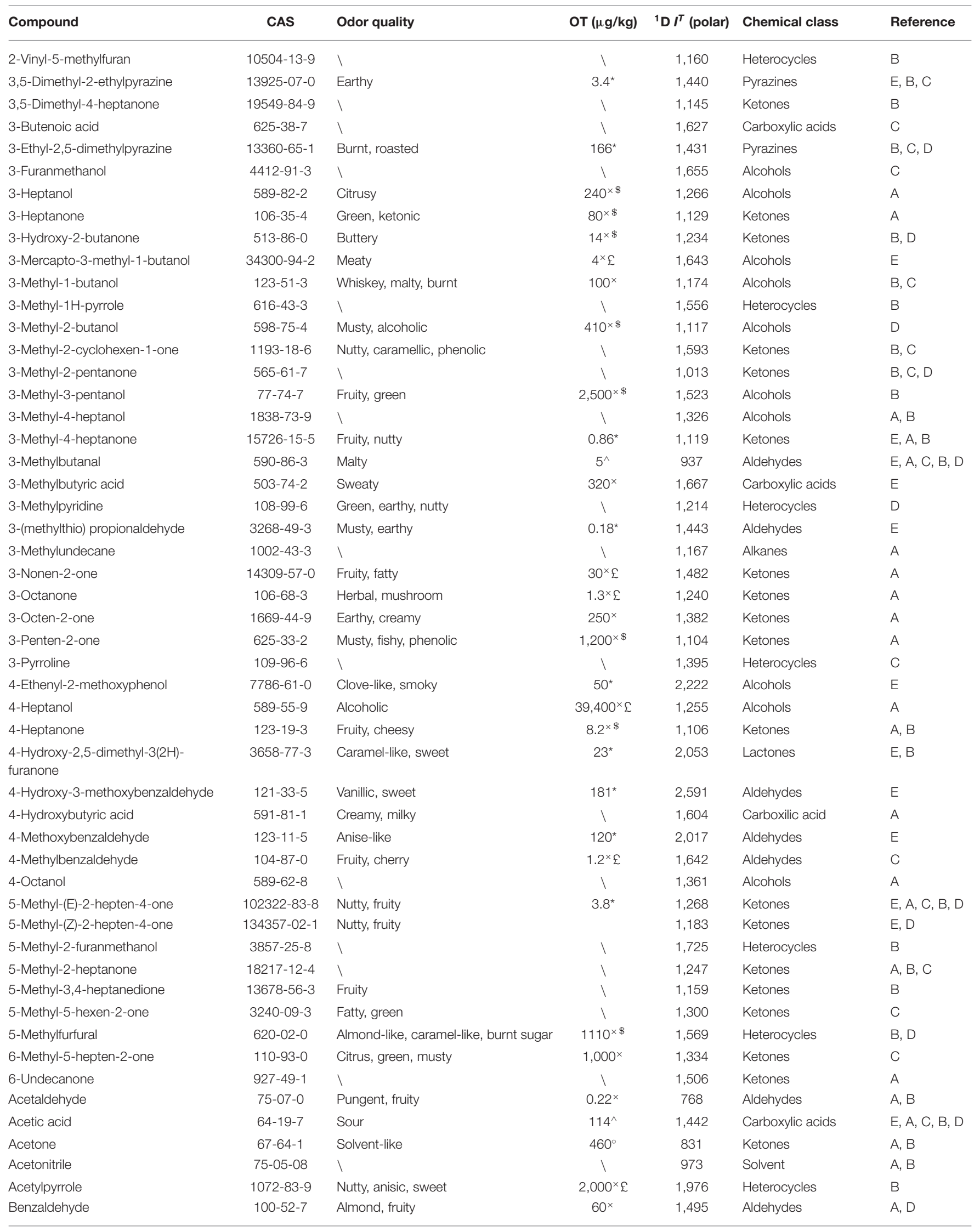


TABLE 5 | Continued

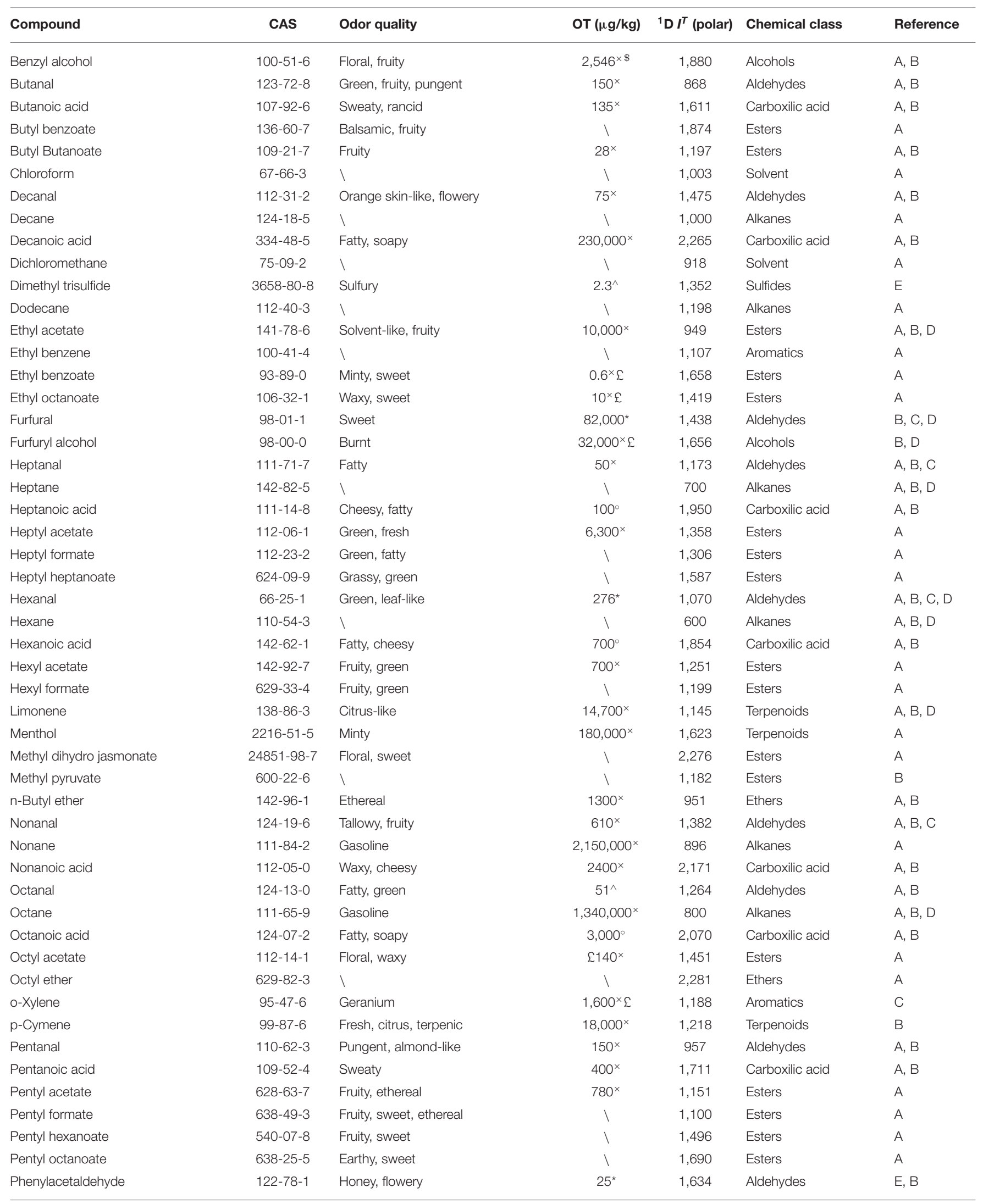


TABLE 5 | Continued

\begin{tabular}{|c|c|c|c|c|c|c|}
\hline Compound & CAS & Odor quality & OT ( $\mu \mathrm{g} / \mathrm{kg})$ & ${ }^{1} \mathrm{D} I^{T}$ (polar) & Chemical class & Reference \\
\hline Phtalide & $87-41-2$ & Sweet, coumarinic & $\backslash$ & 2,317 & Heterocycles & $B$ \\
\hline Propanal & $123-38-6$ & Green, acetaldehyde-like & $9.4^{\times}$ & 820 & Aldehydes & $A$ \\
\hline p-Xylene & $106-42-3$ & Plastic & $250^{\times} £$ & 1,137 & Aromatics & C \\
\hline Pyranone & 28564-83-2 & $\backslash$ & $\backslash$ & 2,236 & Heterocycles & B \\
\hline Pyrazine & $290-37-9$ & Nutty, sweet & 1 & 1,204 & Pyrazines & $D$ \\
\hline Pyridine & $110-86-1$ & Sour, fishy & $920^{x}$ & 1,134 & Heterocycles & $B, D$ \\
\hline Pyrrole & $109-97-7$ & Nutty, sweet, ethereal & $\backslash$ & 1,513 & Heterocycles & $\mathrm{B}, \mathrm{C}, \mathrm{D}$ \\
\hline Sabinene & $3387-41-5$ & Terpenic & $2,000 \times £$ & 1,072 & Terpenoids & $\mathrm{B}$ \\
\hline Sabinene hydrate & $546-79-2$ & Terpenic & $10,000^{\times} £$ & 1,540 & Terpenoids & $\mathrm{B}$ \\
\hline Styrene & $100-42-5$ & Balsamic, almond & $3,100^{x}$ & 1,245 & Aromatics & A \\
\hline Terpinen-4-ol & $562-74-3$ & Spicy, cooling & $590 \times £$ & 1,553 & Terpenoids & $A$ \\
\hline Tetradecane & $629-59-4$ & Mild, waxy & $13,000,000^{x}$ & 1,403 & Alkanes & A \\
\hline Tetrahydrofuran & $109-99-9$ & $\backslash$ & $\backslash$ & 859 & Heterocycles & $A$ \\
\hline Toluene & $108-88-3$ & Sweet & $330^{x}$ & 1,038 & Aromatics & $\mathrm{A}, \mathrm{B}, \mathrm{C}$ \\
\hline trans-4,5-epoxy-(E)-2-Decenal & $134454-31-2$ & Metallic & $25^{x}$ & 1,993 & Aldehydes & E \\
\hline$\alpha$-Pinene & $7785-26-4$ & Herbal, woody & $274^{\times}$ & 1,012 & Terpenoids & $A, C$ \\
\hline$\beta$-Caryophyllene & $87-44-5$ & Sweet, woody & $11,000^{\times} £$ & 1,597 & Terpenoids & C \\
\hline$\gamma$-Butyrolactone & $96-48-0$ & Creamy, oily, fatty & $1,000 \times \$$ & 1,625 & Lactones & $B, D$ \\
\hline$\gamma$-Heptalactone & $105-21-5$ & Sweet, coconut, lactonic & $3,400^{\circ}$ & 1,806 & Lactones & $A, B$ \\
\hline$\gamma$-Hexalactone & $695-06-7$ & Sweet, creamy, lactonic & $8,000^{\circ}$ & 1,662 & Lactones & $A, B$ \\
\hline$\gamma$-Nonalactone & $104-61-0$ & Coconut & $2,400^{\circ}$ & 2,038 & Lactones & $A, B$ \\
\hline$\gamma$-Octalactone & $104-50-7$ & Sweet, coconut, lactonic & $3,500^{\circ}$ & 1,924 & Lactones & $A, B$ \\
\hline$\gamma$-Pentalactone & $129-69-8$ & Herbal, tonka & $\backslash$ & 1,561 & Lactones & $A$ \\
\hline$\gamma$-Terpinene & $99-85-4$ & Terpenic, lime & $55,000^{\times} £$ & 1,241 & Terpenoids & $A, B$ \\
\hline$\Delta$-3-Carene & $13466-78-9$ & Citrus, herbal & $9,300 \times £$ & 1,138 & Terpenoids & $A, C$ \\
\hline$\delta$-Heptalactone & $3301-90-4$ & Coconut, creamy & $\backslash$ & 1,879 & Lactones & A \\
\hline
\end{tabular}

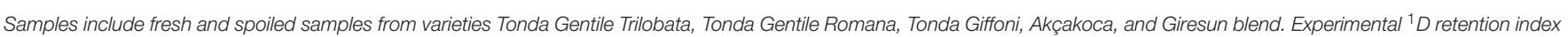

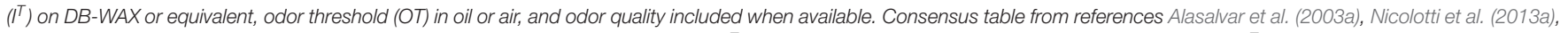

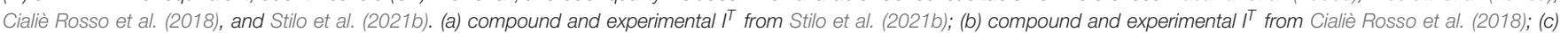

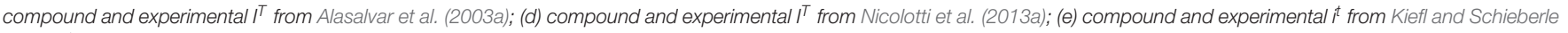

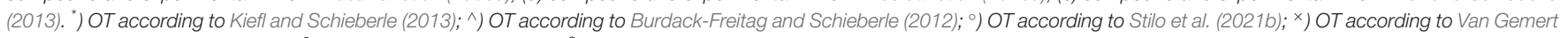
(2003); £) OT reported in air ( $\left.\mu \mathrm{g} / \mathrm{m}^{3}\right)$ when OT in oil not available; ${ }^{\text {) }}$ OT reported in water when OT in oil and air not available.

(Kinderlerer and Johnson, 1992; ÖZDEMIR, 1998; Özdemir et al., 2001; Koyuncu et al., 2005; Locatelli et al., 2015; Ghirardello et al., 2016; Belviso et al., 2017; Memoli et al., 2017; Momchilova et al., 2017; Turan, 2018; Pedrotti et al., 2021), where storage and processing were investigated for their impact on the fatty fraction.

However, recent findings on the evolution of FFAs along with shelf-life (Cialiè Rosso et al., 2021) suggest that post-harvest has a decisive impact on esterases/lipases activity; FFAs are more prone to oxidation than those esterified in TAGs, registering 10 times faster oxidation kinetics (Frega et al., 1999).

Potent odorants profiling indicated the decisive effect of postharvest drying in preserving hazelnut quality and oxidation status; moreover, the marked development of potent odorants with unpleasant notes, formed by autoxidation of essential fatty acids, interestingly evokes the hypothesis that key flavor-related volatiles in vegetable food are generated from essential nutrients and health-promoting components (e.g., amino acids, fatty acids, and carotenoids), while informing the actual nutritional value of the product (Goff and Klee, 2006).

\section{Hazelnut Spoilage Volatiles Signatures}

Hazelnuts quality might be degraded during growing, harvesting, and storage (Giraudo et al., 2018), resulting in some physical and sensorial defects. The industry implements several quality control procedures for incoming batches to check for physical damage: insect-damaged, rotten, twin, and yellowed hazelnut kernels (Mehlenbacher et al., 1993; Caligiani et al., 2014; Belviso et al., 2017; Göncüoglu Taş and Gökmen, 2017; Yuan et al., 2018). Visual inspection by trained staff on representative samples is, nowadays, the quality control procedure of choice in the hazelnut value chain. This approach, highly time-consuming, might be strongly influenced by the level of experience and sensibility of the operator (Giraudo et al., 2018).

Complex carbohydrates undergo enzymatic hydrolysis when attacked by worms, bacteria, or molds, to provide them an available source of metabolic energy. The autocatalytic oxidation of unsaturated fatty acids and the hydrolysis of lipids in free fatty acids are also triggered. These reactions result in a negatively altered aroma and taste, i.e., the rotten defect of the 
fruits (Giraudo et al., 2018). Physical damage, such as dark kernels with white/brown spots, arises from insect bites that transfer saliva enzymes to the nut (e.g., proteinases, esterases, lipases, and amylases). Furthermore, damaged fruit can be more easily attacked by Aspergillus and Penicillium species, the most common fungi found after rainy seasons (Pscheidt et al., 2019), whose metabolic activity might negatively impact the sensory properties of the nut.

Amrein et al. (2010, 2014) identified prenyl ethyl ether (PRE) as the cause of a solvent off-note in ground-haversted hazelnuts. The authors applied a sensory-oriented strategy based on olfactometry coupled to GC (i.e., GC-O), followed by odor value calculation and spiking experiments.

Solid-phase microextraction (SPME) and simultaneous distillation-extraction (SDE) were adopted to extract and isolate volatiles from hazelnut batches showing the solvent off-note. Based on GC-O, performed by a trained panel, an odor active region showing a metallic solvent-like aroma impression was identified. The presence of prenyl ethyl ether was confirmed by GC-MS and retention data.

Quantification results on a series of major volatiles referred that linear saturated aldehydes (hexanal, octanal, and nonanal), reported in many studies as responsible for rancid offnotes, did not show meaningful differences between defective and control samples. Interestingly, in defective samples that reported higher amounts of prenyl ethyl ether, several terpenes (myrcene, limonene, and valencene) were also present in high concentrations.

The authors went ahead to find the possible formation pathway for prenyl ethyl ether. Prenyl alcohols can be formed by Aspergillus, Rhizopus, Penicillium, Eurotium, Mucor, and Fusarium, therefore model experiments with contamination of hazelnuts with the abovementioned molds were conducted, unfortunately without success. To date, it is assumed that this solvent metallic component might be formed as a consequence of mold contamination in presence of unknown co-factors that play a key role in triggering metabolism activation (Amrein et al., 2010, 2014).

Although the cause route of many spoilage defects is still unknown, instrumental methods capable of detecting odorant patterns responsible for perceivable off-odor are of great interest. Moreover, by the implementation of quantitative analytical workflows, quality screening is more objective, and highlyinformative chemical analysis can comprehensively cover many needs, e.g., spoilage detection, rancidity, and authentication markers (Cordero et al., 2010; Kiefl et al., 2012; Cialiè Rosso et al., 2018).

Stilo et al. (2021b) developed an effective strategy to detect odorant patterns in selected spoiled hazelnuts showing perceivable defects. A sensory panel screened by flash profiling (FP) (Dairou and Sieffermann, 2002; Delarue and Sieffermann, 2004) Ordu and Akçakoca samples, harvested in 2015 and 2016, at different shelf-life stages. Samples $(n=29)$ were therefore classified into seven sensory classes: "good quality" ( $O K$ samples) were those eliciting positive attributes and none of the negative attributes arising from the consensus list; "Defected" samples were sub-classified in five different groups based on the predominant off-flavor perceived: Mold, Mold-rancid-solvent, Rancid, Rancid-stale, Rancid-solvent, and Uncoded KO.

Volatiles were extracted and analyzed with high resolution fingerprinting by HS-SPME followed by GC $\times$ GC-TOF MS; 350 untargeted and targeted features were tracked and aligned over all the samples. By unsupervised statistics, i.e., hierarchical clustering based on Pearson correlation, a series of informative volatiles showed a strong correlation with Mold and Moldrancid-solvent classes. Volatile fatty acids (hexanoic, heptanoic, octanoic, and nonanoic acid), lactones $(\gamma$-hexalactone, $\delta$ heptalactone, $\gamma$-heptalactone $\gamma$-octalactone, $\gamma$-nonalactone), 1 nonanol, and 3-nonen-2-one were distinctive and enabled independent clustering of spoiled hazelnuts from good $(O K)$ samples. On the other hand, $O K$ samples were connoted by higher amounts of short-chain linear alcohols (2-pentanol and 2heptanol), butyl ether, butyl benzoate, and 4-heptanone, odorants mainly correlated to positive attributes of balsamic, fruity, and herbal notes.

Results on up- or down-regulation of specific volatiles between spoiled and OK samples are illustrated on histograms in Figure 2. Mold and Mold-rancid-solvent samples were characterized by higher amounts of saturated and unsaturated aldehydes, linear alcohols, and carboxylic acids. Butanal, decanal, and lactones were higher in Mold, while pentanoic acid was more abundant in the Mold-rancid-solvent. In addition, Mold-rancidsolvent class exhibited higher amounts of 3-penten-2-one and 3-octen-2-one, responsible for earthy and musty notes.

The Rancid, Rancid-solvent, and Rancid-stale classes had common chemical patterns: higher amounts of linear saturated aldehydes (from C4 to C14) likely informing about the extent of lipid oxidation probably triggered by differential activation of autocatalytic and enzymatic processes (Kinderlerer and Johnson, 1992; Ghirardello et al., 2016; Cialiè Rosso et al., 2018). Moreover, Rancid-solvent samples were also connoted by the up-regulation (compared to $O K$ samples) of several primary alcohols (1-nonanol, 1-decanol, 1-dodecanol, and 1tetradecanol), and Rancid with higher amounts of 3-penten2-one and 3-undecanone, while Rancid-stale of 2-pentanone and 2-heptanone.

Fatty acids and lactones dominate the differential distribution in Mold and Mold-rancid-solvent samples: these chemical classes are correlated to the fatty acid degradation promoted by different mold genus (e.g. Aspergillus, Penicillium, Rhizopus, Fusarium etc.), developed during post-harvest (Memoli et al., 2017). Lactones are formed by fatty acid hydroxylation in odd or even positions, followed by $\beta$-oxidation and chain reduction. As a function of hydroxyl group position, lactonization results in $\gamma$-, $\delta$ - or $\varepsilon$-lactones (Romero-Guido et al., 2011). Their presence in moldy samples, likely contaminated by fungi, is in keeping with literature data; molds produce lactones by enzymatic catalysis with the degradation of hydroxyl fatty acids; when the native substrate is not available, they implement the hydroxylation step (Romero-Guido et al., 2011) to enable lactones formation. Moreover, molds produce lipolytic enzymes (i.e., lipases and esterase) to promote TAGs hydrolysis resulting in a higher amount of high molecular weight lactones produced by higher homologs released from TAGs (Memoli et al., 2017). 


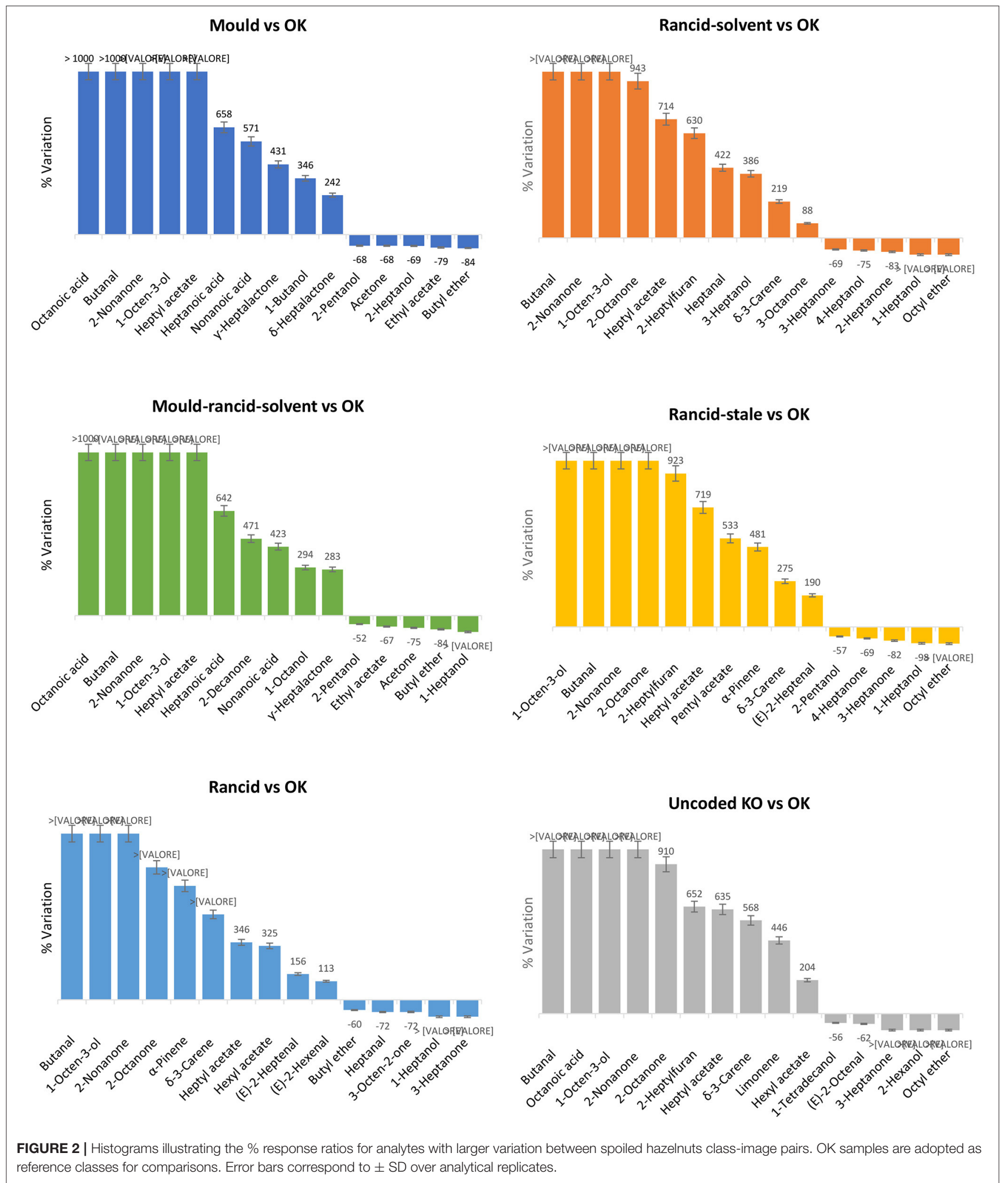

Another critical defect is associated with the bug damage, by species like Palomena prasina L., Gonocerus acuteangulatus G., and Piezodorus lituratus F., determining tissue necrosis and decreasing the overall quality of the hazelnut kernel due to off-flavors and bitterness (Singldinger et al., 2018). Singldinger et al. (2018) identified, through a 
sensory-guided investigation, bitter-tasting diarylheptanoids: asadanin, giffonin $\mathrm{P}$, and other congeners (i.e., (E)-7,9,10,13tetrahydroxy-1,7-bis(2-hydroxyphenyl)hept-9-en-11-one; 4,12,16-trihydroxy-2-oxatricyclo[13.3.1.13,7]-nonadeca1(18),3,5,7(20),8,15,17-heptaen; 2-(3-hydroxy-2-oxoindolin-3yl) acetic acid $3-\mathrm{O}-6^{\prime}$-galactopyranosyl-2" $-\left(2^{\prime \prime}\right.$ oxoindolin $\left.-3^{\prime \prime} \mathrm{yl}\right)$ acetate and $3-(\mathrm{O}-\beta-\mathrm{d}$-glycosyl $)$ dioxindole-3-acetic acid) with bitter and astringent taste qualities in cimiciato-infected hazelnuts. Although non-volatiles, these sensory active compounds have a detrimental effect on hazelnut sensory quality and might also exert some sensorial synergy with potent odorants responsible for aroma off-notes.

\section{POTENT ODORANTS AND KEY-AROMA COMPOUNDS RESPONSIBLE FOR HAZELNUTS AROMA QUALITY}

In the conceptualization of molecular sensory science, i.e., sensomics (Schieberle and Hofmann, 2011), key-odorants are those odor active compounds whose amount in the sample exceeds their OT $(\mathrm{OAV}>1)$, and for which omission in aroma recombination experiments confirm the key-role in eliciting the distinctive aroma, i.e., the aroma blueprint, of the original product. It has to be noted that recent findings did not exclude that so-called interferents, i.e., volatiles with lower or marginal odor activity, might play a role in modulating key-odorants perception while contributing to the overall aroma (Charve et al., 2011; Cordero et al., 2019; Bressanello et al., 2021).

In keeping with these observations, the next paragraphs report major research findings dealing with the identification of potent odorant patterns in raw and roasted hazelnuts. By literature selection, sensory-driven investigations were prioritized because of the intrinsic biological validation they offer concerning the complex phenomenon of olfactory perception.

\section{Raw Hazelnuts Aroma}

Raw hazelnut aroma elicits some specific notes as reported by sensory experiments (descriptive sensory analysis - DSA and quantitative descriptive analysis - QDA): fruity, fatty, nutty, green, rancid, citrus-like, earthy, flowery, malty, popcorn-like, potato-like, sour, woody, and phenolic (Burdack-Freitag and Schieberle, 2010; Alasalvar et al., 2012a).

These odor notes were explored by Alasalvar et al. (2003a, 2010, 2012a) in many different cultivars grown in Turkey (Aci, Cavcava, Çakildak, Foşa, Ham, Incekara, Kalinkara, Kan, Karafindik, Kargalak, Kuş, Mincane, Palaz, Sivri, Tombul, Uzunmusa, Yassi Badem, and Yuvarlak Badem). By correlating DSA profiles, GC-O results, and quantitative data on chemical patterns (Alasalvar et al., 2003a), the role of some potent odorants eliciting characteristic aroma was established. However, without the validation step by recombination and omission experiments, it was not possible to define key-aroma compounds (Dunkel et al., 2014) over the group of odorants.

The application of sensomic concepts was successful in this direction, Burdack-Freitag and Schieberle (2010, 2012), Kiefl and Schieberle (2013), and Kiefl et al. (2013) unraveled the aroma code of hazelnuts (raw and roasted) by focusing on different cultivars/origins. They covered the Italian production by studying Tonda Gentile Romana (Lazio, Italy), Tonda Gentile Trilobata (Piemonte, Italy), Tonda Giffoni (Campania, Italy), and Turkey high-quality blend from the Akçakoca region.

Burdack-Freitag and Schieberle (2010, 2012) pre-screened potent odorants by GC-O, performed as aroma extract dilution analysis (AEDA). The distillate/extract obtained by solventassisted flavor evaporation (SAFE) of raw hazelnuts extracts, was further fractionated in a neutral-basic fraction (NBF) and the acidic fraction (AF). In the NBF, 37 odor-active zones were detected with a flavor dilution factor (FD) range of 44096. Six odorants dominated for their prevalence in the SAFE extracts screening: 2-methoxy-3-isopropylpyrazine elicited an intense bell pepper-like odor; 5-methyl-4-heptanone had a fruity hazelnut-like note; 2-methoxy-3,5-dimethylpyrazine showed an earthy odor; ethyl 2-methylbutanoate, with a fruity note; 5methyl-(E)-2-hepten-4-one with fruity, hazelnut-like aroma; and 2-methoxy-3-isobutylpyrazine showing a bell pepper-like attribute. Interestingly, the fruity hazelnut-like ketone, 5-methyl4-heptanone, was characterized for the first time in raw hazelnuts: its very low OT (i.e., $0.05 \mu \mathrm{g} / \mathrm{L}$ in water) is comparable to that of the nutty ketone, 5-methyl-(E)-2-hepten-4-one (i.e., filbertone).

High relevance was also confirmed for linalool, (E)- $\beta$ damascenone, and 4-methylphenol (flowery, boiled apple-like, and smoky notes respectively). Within the acidic fraction, acetic acid (sour), 2- and 3-methylbutanoic acid (sweaty), and 4-hydroxy-2,5-dimethyl-3(2H)-furanone (caramel-like) were identified.

Accurate quantification by stable isotope dilution analysis (SIDA) (Burdack-Freitag and Schieberle, 2012) identified, within odorants screened by GC-O and AEDA, those exceeding their OT (i.e., OAV > 1). They are listed in Table 6; among them, in raw hazelnuts linalool, 5-methyl-4-heptanone, and 2-methoxy-3,5-dimethylpyrazine showed OAVs $>100$ likely dominating the overall aroma of raw kernels. An aroma recombinant including all odorants with $\mathrm{OAV}>1$ conferred a quite high similarity to the aroma profile of raw hazelnuts, with fruity-nutty, fatty, popcorn-like, flowery, bell-pepper-like, malty, and potato-like considered almost equivalent to the natural aroma profile.

Kiefl and Schieberle (2013) and Kiefl et al. (2013) further explored the aroma code of hazelnuts by extending the investigation to high-quality cultivars from the two major production areas (Turkey and Italy). Additional odorants, with high OAVs, characterizing raw hazelnuts were identified in 2-propionyl-1-pyrroline, 2-acetyl-1,4,5,6-tetrahydropyridine, and 2-acetyl-3,4,5,6-tetrahydropridine all eliciting popcorn-like, roasty notes.

Sensomic studies on hazelnut sensometabolome (Kiefl, 2013), inspired the definition of a performance parameter known as Limit of Odor Activity Value (LOAV), defined as the ratio of the respective OT and the analytical limit of quantification (LOQ). This concept, applied to aroma compounds of Tonda Gentile Trilobata hazelnuts, enabled the evaluation of the method's ability in detecting all key odorants screened by 
TABLE 6 | Key-aroma compounds are defined through the sensomics approach in raw and roasted hazelnut samples from cultivars Tonda Gentile Trilobata (G), Tonda Gentile Romana (R), and Akçakoca blend (A).

\begin{tabular}{|c|c|c|c|c|c|c|c|}
\hline Aroma compound & Aroma quality & $\begin{array}{c}\text { OAV } \\
(\operatorname{raw} R)^{a, b}\end{array}$ & $\begin{array}{c}\text { OAV } \\
\text { (roasted R) }^{\mathrm{a}, \mathrm{b}}\end{array}$ & $\begin{array}{c}\text { OAV } \\
(\operatorname{raw} G)^{b}\end{array}$ & $\begin{array}{c}\text { OAV } \\
\text { (roasted G) }^{\mathrm{b}}\end{array}$ & $\begin{array}{c}\text { OAV } \\
(\operatorname{raw} A)^{\mathrm{b}}\end{array}$ & $\begin{array}{c}\text { OAV } \\
(\text { roasted A })^{\mathrm{b}}\end{array}$ \\
\hline$(E, E)-2,4$-Decadienal & Deep-fried & $<1$ & 7 & $<1$ & 1 & 8 & 6 \\
\hline$(E, E)-2,4-$ Nonadienal & Deep-fried & 5 & 6 & $<1$ & 1 & $<1$ & 1 \\
\hline (Z)-2-Nonenal & Fatty & 1 & 300 & 1 & $\backslash$ & $\backslash$ & $\backslash$ \\
\hline (Z)-2-Octenal & Fatty & $<1$ & 2 & 1 & 1 & 1 & 1 \\
\hline 2,3-Butanedione & Buttery & $<1$ & 85 & $\backslash$ & $\backslash$ & $\backslash$ & $\backslash$ \\
\hline 2,3-Diethyl-5-methylpyrazine & Musty, nutty & 9 & 266 & 9 & 204 & 9 & 103 \\
\hline 2,3-Pentanedione & Buttery & $<1$ & 1,140 & 1 & $\backslash$ & $\backslash$ & 1 \\
\hline 2-Acetyl-1,4,5,6-tetrahydropyridine & Caramel & 46 & 229 & 46 & 219 & 46 & 81 \\
\hline 2-Acetyl-1-pyrroline & Popcorn-like & 13 & 470 & 24 & 599 & 24 & 308 \\
\hline 2-Acetyl-3,4,5,6-tetrahydropyridine & Roasted, caramel & 36 & 184 & 36 & 132 & 36 & 36 \\
\hline 2-Furfurylthiol & Coffee-like & 4 & 47 & 8 & 8 & 8 & 8 \\
\hline 2-Methoxyphenol & Phenolic, smoky & $<1$ & 2 & 1 & 1 & 1 & 1 \\
\hline 2-Methylbutanal & Malty & $<1$ & 36 & $\backslash$ & $\backslash$ & $\backslash$ & 1 \\
\hline 2-Phenylacetaldehyde & Green, floral & $<1$ & 46 & $<1$ & 48 & $<1$ & 41 \\
\hline 2-Propionyl-1-pyrroline & Popcorn-like & 11 & 168 & 22 & 243 & 22 & 113 \\
\hline 2-Thenylthiol & Coffee-like & $<1$ & 27 & 1 & $\backslash$ & 1 & 1 \\
\hline 3-(Methylthio)-propanal & Cooked potato & 13 & 45 & 1 & $\backslash$ & $\backslash$ & $\backslash$ \\
\hline 3-(Methylthio)propionaldehyde & Musty, earthy & 15 & 620 & 15 & 442 & 15 & 464 \\
\hline 3,5,5-Trimethyl-2(5H)-furanone & Seasoning-like & $<1$ & 2 & 1 & $\backslash$ & 1 & 1 \\
\hline 3,5-Dimethyl-2-ethylpyrazine & Burnt, roasted & 1 & 25 & 1 & 11 & 1 & 11 \\
\hline 3-Ethyl-2,5-dimethylpyrazine & Burnt, roasted & $<1$ & 3 & $<1$ & 2 & $<1$ & 1 \\
\hline 3,7-Dimethylocta-1,6-dien-3-ol & Citrus, floral & 12 & 12 & 12 & 12 & 12 & 12 \\
\hline 3-Methyl-4-heptanone & Fruity, nutty & 218 & 81 & 126 & 143 & 93 & 91 \\
\hline 3-Methylbutanal & Malty & 7 & 1,330 & 1 & 1 & $\backslash$ & 1 \\
\hline 3-Methylbutanoic acid & Sour, sweaty & 2 & 8 & 1 & 4 & 1 & 3 \\
\hline $\begin{array}{l}\text { 4-Hydroxy-2,5-dimethyl-3(2H)- } \\
\text { furanone }\end{array}$ & Caramel & $<1$ & 89 & 1 & 79 & 1 & 45 \\
\hline 4-Hydroxy-3-methoxybenzaldehyde & Vanillic, sweet & $<1$ & 4 & $<1$ & 2 & $<1$ & 1 \\
\hline 5-Methyl-(E)-2-hepten-4-one & Nutty, fruity & 2 & 60 & 2 & 130 & 2 & 71 \\
\hline Acetic Acid & Sour & 3 & 8 & $\backslash$ & $\backslash$ & $\backslash$ & 1 \\
\hline Dimethyl trisulfide & Sulfurous, cabbage & $<1$ & 84 & $<1$ & 1 & 8 & 6 \\
\hline Hexanal & Green & 5 & 5 & 1 & $\backslash$ & $\backslash$ & $\backslash$ \\
\hline Octanal & Fatty, soapy & 3 & 28 & $\backslash$ & $\backslash$ & $\backslash$ & $\backslash$ \\
\hline
\end{tabular}

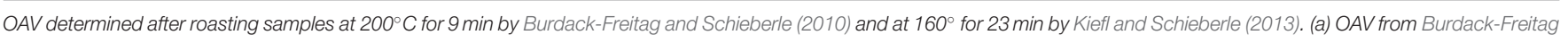
and Schieberle (2010); (b) OAV from Kiefl et al. (2013).

GC-O and AEDA. In particular, in the reference study (Kiefl et al., 2013), authors were able to quantify 30 potent odorants by SIDA with GC $\times$ GC-TOF MS, although just 15 of them achieved a LOAV $\geq 1$, meaning that the method was unable to assign the appropriate relevance (i.e., keyodorant ranking) to some analytes present at a sub-ppb level. Examples are 2-isopropyl-3-methoxypyrazine, eliciting green bell pepper-like notes that showed a LOAV $=0.02$ and 2acetyl-1-pyrroline with a roasty popcorn-like smell, with a $\mathrm{LOAV}=0.04$.

\section{Roasted Hazelnuts Aroma}

While raw hazelnuts are characterized by a general weak aroma (Kiefl and Schieberle, 2013), the roasting process enhances many existing odor notes (e.g., nutty-fruity, sweet-caramel, and malty) by triggering several reactions on non-volatile precursors and primary metabolites, while generating new, yet intense, sensations like roasty, pop-corn like, and coffee-sulfuric (Kiefl and Schieberle, 2013).

The chemical reactions triggered by the thermal treatment produces common chemical patterns in many foods; Dunkel et al. (2014) for example, revising the combinatorial odor codes of many foods, identified a strong network structure based on processing technologies (e.g., dry-roasting, fermentation, etc.). Volatiles patterns and odor codes of dry thermally processed foods (roasted, deep-fried, baked) have in common many traits due to the activation of the Maillard reaction (Hofmann and Schieberle, 1998; Van Boekel, 2006; Göncüoglu Taş and Gökmen, 
2017), which occurs between reducing sugars and amino acids, and sugars degradation (i.e., caramelization).

As an example, alkyl-pyrazines, characterized by low odor thresholds for humans (e.g., 0.007-0.018 $\mu \mathrm{g} \mathrm{L}-1$ in water), are responsible for the earthy and baked potato-like odors; of them, 2-ethyl-3,5-dimethylpyrazine, 2-ethenyl-3,5-dimethylpyrazine, and 2,3-diethyl-5-methylpyrazine are frequently detected as KFOs in thermally processed foods such as for example, roasted coffee (Blank et al., 1992), French fries (Grosch, 2001), chocolate (Schnermann and Schieberle, 1997), cocoa (Frauendorfer and Schieberle, 2008; Magagna et al., 2018), and peanuts (Chetschik et al., 2010).

In hazelnuts, the complex reaction framework of Maillard reaction, arising by the intersection of many pathways, generates key-odorants impacting on hazelnuts' aroma. They are alkyl methoxy-pyrazines (e.g., roasty, 3,5-dimethyl-2-ethylpyrazine/ earthy; 2,3,5-trimethylpyrazine/earthy; 2,3-diethyl-5methylpyrazine/earthy; 2-isopropyl-3-methoxypyrazine/ pea-like and green pepper-like); Strecker aldehydes (2- and 3-methylbutanal/malty; phenylacetaldehyde/flowery and honeylike); ketones (2,3-butanedione and 2,3-pentanedione/buttery; 3methyl-4-heptanone/fruity and nutty); and heterocycles formed by deoxyosones dehydration in presence of ammonia (e.g., 4hydroxy-2,5-dimethyl-3(2H)-furanone/caramel-like and sweet; 2-acetyl-1-pyrroline and 2-propionyl-1-pyrroline/popcorn-like, roasty) (Alasalvar et al., 2004; Seyhan et al., 2007; Burdack-Freitag and Schieberle, 2012; Kiefl and Schieberle, 2013). Unpleasant notes generated by thermal reactions are those elicited by alkylpyridines generally connoted by burnt and astringent aroma (Van Boekel, 2006).

The role of roasting was the object of many studies aimed at a better understanding of optimal conditions responsible for the generation of pleasant aroma, crunchy texture, and color (Saklar et al., 2001, 2003; Alasalvar et al., 2003a; Alamprese et al., 2009; Cordero et al., 2010; Ciarmiello et al., 2013; Donno et al., 2013; Kiefl and Schieberle, 2013; Belviso et al., 2017; Artik et al., 2021). It was demonstrated that cultivar/origin and storage conditions along shelf-life had a great impact on volatiles generation. Nicolotti et al. (2013a) compared volatiles fingerprints from industrially roasted hazelnuts (Tonda Gentile Trilobata - Piedmont Italy and Ordu - Turkey) to those obtained in lab-scale with specific time/temperature profiles. Authors identified robust markers of roasting namely 5-methylfurfural, 1(H)-pyrrole, furfuryl alcohol, 1(H)-pyrrole-2-carboxaldehyde, 1-hydroxy-2-propanone, dihydro-2(3H)-furanone, acetic acid, pyridine, furfural, pyrazine, and several alkyl-pyrazines; and related indices/ratios whose $\%$ increment along roasting kinetic showed great stability among cultivars 5-methylfurfural/ 2,5-dimethylpyrazine; 5-methylfurfural/2-methylpyrazine; and 2,5-dimethylpyrazine/2,3-dimethyl-pyrazine. Interestingly, the key-odorant 5-methyl-(E)-2-hepten-4-one showed a strong cultivar/origin-related increment with Italian Tonda Gentile Trilobata showing an early increase of this potent odorant at mild roasting conditions.

The application of sensomics to roasted hazelnuts unrevealed their aroma blueprint; studies are indicating the presence of some generalist key-odorants in combination with others with a more distinctive and peculiar impact on the overall perception (i.e., individualists) (Dunkel et al., 2014).

Burdack-Freitag and Schieberle (2010, 2012) for Tonda Gentile Romana hazelnut paste validated the pre-eminent role of several key-odorants here listed in descending order of OAV: 3-methylbutanal/malty/OAV 1330; 2,3pentanedione/caramel/OAV 1140; 2-acetyl-1-pyrroline/popcornlike/OAV 360; (Z)-2-nonenal/tallow/OAV 300; dimethyl trisulfide/sulfury, cabbage/OAV 164; 2-furfurylthiol/coffeelike/OAV 86; 2,3-butanedione/buttery/OAV 85; 4-hydroxy2,5-dimethyl-3(2H)-furanone/caramel/OAV 77; 5-methyl-4heptanone/nutty, fruity/OAV 66; 3-(methylthio)propanal/cooked potato/OAV 45; 2-methylbutanal/malty/OAV 36; octanal/soapy/OAV 28; 2-thenylthiol/coffee-like/OAV 27; 2-ethyl-3,5-dimethylpyrazine/roasted potato/OAV 21; 5-methyl-(E)-2-hepten-4-one/nutty, fruity/OAV 13; $(E, E)$ 2,4-nonadienal/deep-fried/OAV 11; (E,E)-2,4-decadienal/deepfried/OAV 10.

Later, Kiefl and Schieberle (2013) and Kiefl et al. (2013), extended the knowledge about high-quality hazelnuts aroma blueprint by elucidating the role of several additional odorants all present at OAVs $>$ 1. They were: 2,3-diethyl-5-methylpyrazine/earthy, roasty; 2-acetyl-3,4,5,6-tetrahydropyridine/popcornlike, roasty; phenylacetaldehyde/honey, flowery; 2-propionyl-1-pyrroline/popcorn-like, roasty; 4hydroxy-3-methoxybenzaldehyde/vanillic, sweet; and dimethyl trisulfide/sulfury.

Moreover, by applying sensomic principles on roasting kinetics (Kiefl et al., 2012; Kiefl and Schieberle, 2013), further insights on the hedonic role of odorant patterns were added. Hazelnuts were submitted to lab-scale roasting in a ventilated oven at $160^{\circ} \mathrm{C}$ for 12,23 , or $30 \mathrm{~min}$. Cultivars/origin selected were Tonda Gentile Trilobata (G) from Piedmont, Italy; Tonda Gentile Romana (R) from Lazio, Italy; Tonda Giffoni (Gi) from Campania, Italy; Akçakoca blend from Turkey.

Sensory tests were conducted with a trained panel with 24 judges (details in Kiefl and Schieberle, 2013). A QDA and projective mapping experiments were performed on raw and 23 min. roasted samples to evaluate similarities and differences among samples. QDA evaluated the aroma intensity of eight attributes (coffee-like, sulfury; nutty, fruity; smoky, phenolic; malty; sweet, caramel-like; roasty, popcorn-like; fatty; earthy, green) by a seven-point scale in a range between $0-3$. For the projective mapping, panelists were instructed as follows: "Two samples should be placed very near if they seem identical, and two samples should be placed distant to one another if they seem different to you; this should be done according to your own criteria; do not hesitate to express strongly the differences you perceive by using the most part of the screen (total space)" (Kiefl and Schieberle, 2013).

Aroma profiles of raw hazelnuts, as shown by the spidergraph resulting from the QDA (Figure 3A), were very similar, as also stated by other authors (Seyhan et al., 2007). On the other hand, roasted samples showed some meaningful intensity differences. The roasted Tonda Gentile Trilobata from Piedmont $(\mathrm{G})$, reported weaker coffee-like and roasty odor notes 
(Figure 3B) accompanied by an intense nutty perception. However, by comparing concentration profiles for key-odorants among all analyzed samples (Table 4), sensory differences and odorant patterns could not be easily correlated.

Some analytes, most sensitive to roasting conditions (Kiefl et al., 2012; Nicolotti et al., 2013a), had a 100-fold increase from raw (0) to $30 \mathrm{~min}$; They were identified as: 5-methyl-(E)-2-hepten-4-one; 2-acetyl-1-pyrroline; 2propionyl-1-pyrroline; 2-acetyl-1,4,5,6-tetrahydropyridine; 2-acetyl-3,4,5,6-tetrahydropyridine; 2-acetylpyridine,3,6dimethyl-2-ethylpyrazine; 3-(methylthio)propionaldehyde; 2-phenylacetaldehyde; 3,5-dimethyl-2-ethylpyrazine; and 2,3-diethyl-5-methylpyrazine.

Projective mapping experiments showed great correlation, as a primary variable, to the roasting degree, resulting in three main clusters defined by raw hazelnuts, mild-to-optimal roasting, and over-roasting conditions. Moreover, some samples were classified into separate groups. Assessors intuitively ordered samples according to the roasting degree along the first dimension (i.e., horizontal axis) of the project map while using the second dimension (i.e., the vertical axis) to discriminate hazelnuts by a hedonic scale. Further experiments, recombining odorant patterns for optimally roasted hazelnuts in a sunflower oil medium, confirmed the pre-eminent role of identified key odorants and suggested some synergies and suppression phenomena between them.

The perceptual pattern obtained with aroma recombinants shared a high degree of similarity with those obtained by real samples. Authors stated that eight key-odorants were fundamental to reconstruct the hazelnut aroma blueprint: 3-methyl-4-heptanone elicits the weak nutty aroma of raw hazelnuts, while the pattern of 5-methyl-(E)2-hepten-4-one, 2-acetyl-1-pyrroline, 2-propionyl-1-pyrroline, 3,6-dimethyl-2-ethylpyrazine, 3,5-dimethyl-2-ethylpyrazine, 2,3-diethyl-5-methylpyrazine elicits a nutty, roasty aroma; high amounts of 2-furfuryl mercaptan impart roasty and coffee-like odor in over-roasted samples.

Accurate quantification of aroma compounds by SIDA, or suitable approaches (Sgorbini et al., 2019), combined with GC $\times$ GC-TOF MS proved to be highly effective to decode the aroma blueprint at a molecular level due to the very low LOAVs achievable (Kiefl et al., 2013; Nicolotti et al., 2013b). However, besides the distinctive patterns of key odorants that clearly evoke hazelnut aroma qualities, to date researchers did not find any other volatile capable of consistently explaining hedonic differences across different cultivars. Sensoryoriented strategies, accompanied by high-resolution separations (i.e., multidimensional analytical approaches) and data mining represent the ultimate solution to unravel the combinatorial code of olfaction behind food sensory perception.

The role of so called interferent volatiles (Cordero et al., 2019) should be better elucidated since they can modulate odorant pattern perception, enabling differential activation of the Receptor Code. Moreover, the presence of chiral odorants in enantiomeric excess or distinctive ratios might influence the hedonic profile; for example, 5-methyl-(E)-2-hepten-4-one (i.e., filbertone) enantiomers are characterized by different odor qualities and OTs. The next paragraph reports some insights on filbertone chiral recognition and functional properties.

\section{Filbertone as Hazelnuts Individualist Key-Odorant}

The molecule configuration is crucial in determining its aroma perception: enantiomers may differ in the aroma intensity, as it is the case of menthol and camphor, or even in the flavor itself, as it is for 3-methylthiobutanal (i.e., methional) where the $(R)$ configured molecule elicits the typical odor of cooked potatoes, while the (S)-configured stereoisomer is odorless (Weber and Mosandl, 1997; Zawirska-wojtasiak, 2006). Such characteristic is fundamental with hazelnuts too since filbertone (i.e., 5-methyl(E)-2-hepten-4-one), the key-odorant contributing to the nutty aroma (Jauch et al., 1989; Alasalvar et al., 2004; Burdack-Freitag and Schieberle, 2012; Kiefl et al., 2013), might be present as $R$ or $S$ enantiomer(s) on the chiral center on C5 (Puchl'ová and Szolcsányi, 2018).

Filbertone amounts greatly vary among cultivars, higher levels were reported for Tonda Gentile Trilobata cultivar independently by harvest area (Piedmont Italy vs. Georgia) (Cialiè Rosso, 2020), justifying the fact that this cultivar is particularly appreciated by consumers and defined as the gold standard in the confectionery industry.

Jauch et al. (1989) were the first that effectively discriminated by enantioselective GC (ES-GC) the $R$ - and $S$-filbertone while also describing marked olfactory differences in terms of odor intensity and quality, with the $S$ - enantiomer characterized by metallic, fatty, and pyridine perceptions, while the $R$ - one by buttery and chocolate-like notes; moreover, the $R$ - enantiomer has a 10 -fold lower odor threshold. The $R$ - and $S$ - enantiomers are not equally abundant in the kernel: Ruiz del Castillo et al. (2003) investigated the enantiomeric distribution in both raw and roasted hazelnuts obtaining between 70 and $90 \%$ of enantiomeric excess $(e e)$ for the $S$-filbertone depending on the variety regarding raw hazelnuts (e.g., Turkish hazelnuts showed $54-56 \%$ ee, whereas Italian ones revealed 62-63\% ee) (Jauch et al., 1989). Roasted samples, instead exhibited an ee of only $17 \%$ for the $S$-enantiomer. Nevertheless, the filbertone concentration increased by 35 -fold during roasting. The differential increase of the R-enantiomer during roasting could be likely due to a thermal pathway (Güntert et al., 1991; Blanch and Jauch, 1998) whose precursors are still unknown.

\section{CORRELATIONS BETWEEN PRIMARY METABOLITES AND AROMA COMPOUNDS: THE CONCEPT OF AROMA POTENTIAL}

The concept of aroma potential was firstly introduced by Cialiè Rosso et al. (2018) in a study focused on hazelnut volatilome evolution along with shelf-life. The concept arose by the observation that post-harvest drying conditions appeared fundamental to inactivate exogenous and endogenous enzymes, providing more stable kernels throughout their shelf-life, independently of storage conditions (e.g., atmosphere 
A

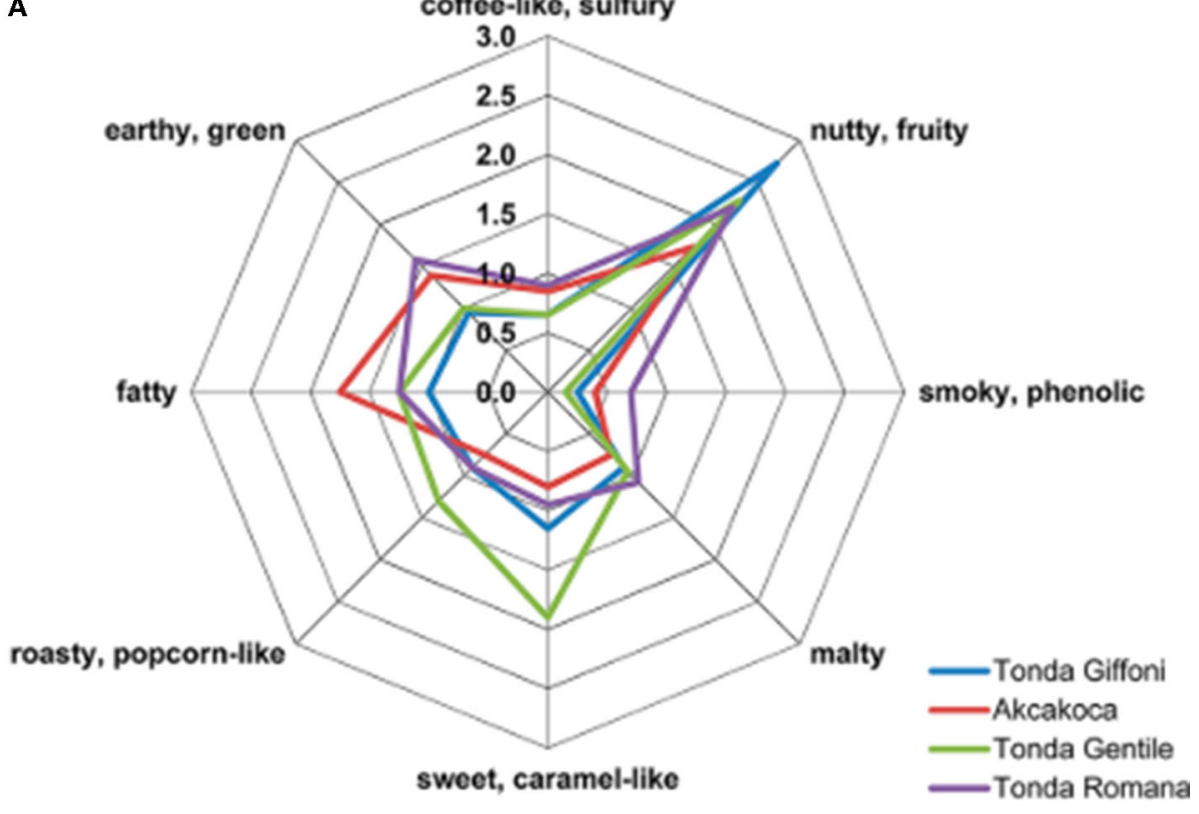

B

B coffee-like, sulfury

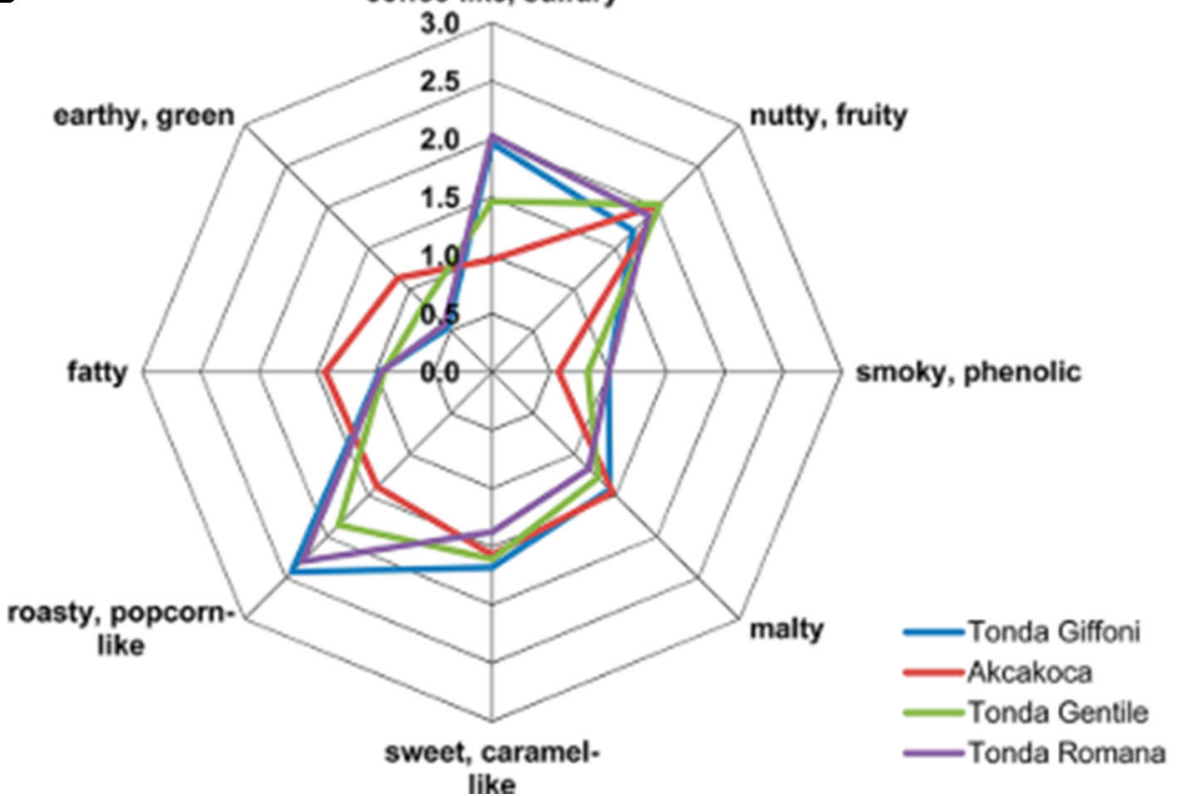

FIGURE 3 | Qualitative sensory analysis results illustrated as spider graphs and corresponding to four hazelnuts cultivars/blends as raw (A) and roasted (B) [23 min at $\left.160^{\circ} \mathrm{C}\right]$ kernels. Figure from Kiefl and Schieberle (2013).

composition, temperature, and time). Volatile patterns evolution indicated that lipid oxidation and spoilage occurred more decisively on those samples exposed to a less efficient drying (i.e., sun-drying $v s$. industrial drying at low temperatures), stored at normal atmosphere and ambient temperature.

Researchers analyzed volatiles patterns from hazelnuts roasted at lab-scale $\left(160^{\circ} \mathrm{C}-15 \mathrm{~min}\right)$ after storage in specified conditions (see Section Effect of Post-Harvest and Storage on Volatilome
Signatures) (Cialiè Rosso et al., 2018). Secondary products of hydroperoxide cleavage (i.e., linear saturated aldehydes -from C5 to C10-, unsaturated aldehydes - (E)-2-heptenal, (E)-2 octenal ad (E)-2 decenal, short-chain fatty acids - pentanoic, octanoic, and nonanoic acid, and linear alcohols - from C5 to C8) were close to the method LOD in freshly roasted hazelnuts (T0) or in those stored in a modified atmosphere. An increasing trend over storage time was also observed for kernels stored in a 

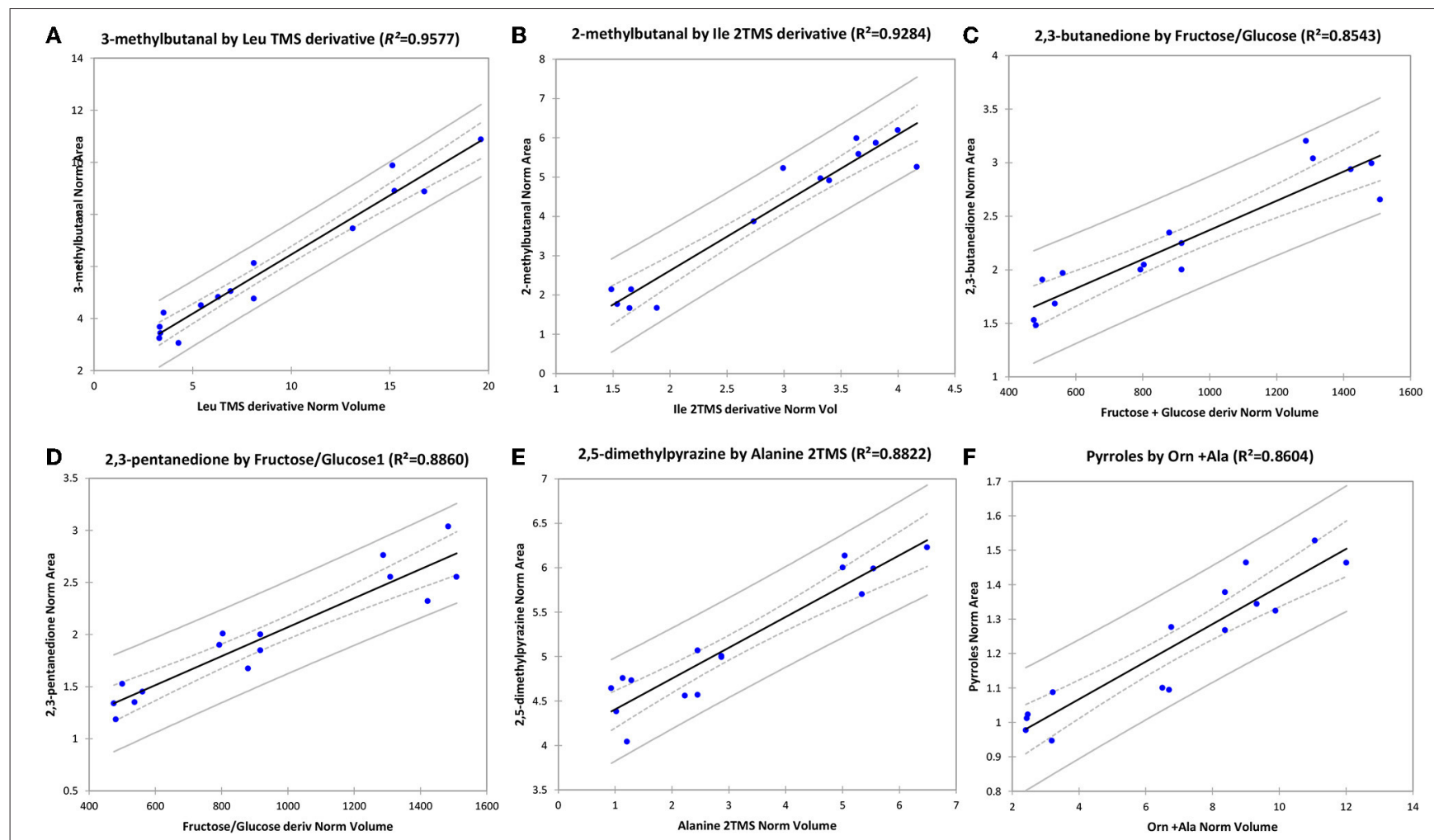

FIGURE 4 | Linear regression functions (confidence interval 95\%) between primary metabolites and/or precursors with corresponding key-odorants in roasted kernels. (A) 3-Methylbutanal with Leucine ( $R^{2}$ 0.9577), (B) 2-Methylbutanal and Isoleucine ( $R^{2}$ 0.9284), (C) 2,3-Butanedione and (D) 2,3-Pentanedione and the sum of fructose (syn- and anti-forms) and glucose (glucopyranose and glucose) ( $R^{2} 0.8543$ and 0.8860), (E) 2,5-Dimethylpyrazine and Alanine ( $\left.R^{2} 0.8822\right)$, and (F) pyrroles and the sum of Ornithine and Alanine ( $R^{2}$ 0.8604) (from Cialiè Rosso et al., 2020).

normal atmosphere as a function of temperature $\left(5\right.$ or $\left.18^{\circ} \mathrm{C}\right)$, as additional stress factors.

Of interest was the trend for some key-odorants responsible for the malty and buttery (2- and 3-methylbutanal, 2,3butanedione, and 2,3-pentanedione), earthy (methylpyrazine, 2-ethyl-5-methyl pyrazine, and 3-ethyl-2,5-dimethyl pyrazine) and caramel-like (2,5-dimethyl-4-hydroxy-3(2H)-furanone) and musty (acetyl pyrrole) notes. Storage at lower temperatures $\left(5^{\circ} \mathrm{C}\right)$ and low-temperature drying preserved their amount in both cultivars/origin (i.e., Tonda Gentile Romana and Ordu) along with shelf-life. The authors argued that there was a fairly stable distribution of their precursors along with shelf-life.

These results suggested the correlation of non-volatile precursors with potent odorants characterizing roasted hazelnut aroma; as for lipid fraction, degradation reactions and kernel viability would have had an impact on primary metabolites known to form under roasting conditions key-aroma substances.

The Pearson correlation coefficient $(r)$ was adopted to evaluate positive or negative correlations between primary metabolites and key-informative volatiles. The study applied advanced fingerprinting strategies combining untargeted and targeted features information from GC $\times$ GC-TOF MS analyses of hazelnut polar extracts followed by oximation-silylation on amino acids, reducing sugars, polyols, and organic acids (Cialiè
Rosso et al., 2020, 2021). Samples analyzed were from Tonda Gentile Trilobata, Tonda Gentile Romana, and Ordu.

Results showed good correlations ( $p$-values $<0.05)$ within primary metabolites, also indicating that Tonda Gentile Trilobata and Tonda Gentile Romana samples showed a higher amount of some non-volatile precursors and primary metabolites compared to the Ordu blend. On the other hand, interesting correlations were established between primary metabolites and volatiles eliciting aroma qualities. These correlations were further explored and tested for their linearity. The coefficients of determination $\left(R^{2}\right)$ of the linear regression were estimated for the relation between the precursor(s) as the independent variable $(x)$ and key-volatiles as the dependent variable $(y)$. Figure 4 reports regression functions between 3-methylbutanal and leucine-Leu $\left(R^{2} 0.9577\right), 2$-methylbutanal and IsoleucineIle $\left(R^{2}\right.$ 0.9284), 2,3-butanedione and 2,3-pentanedione and fructose/glucose derivatives $\left(R^{2} 0.8543\right.$ and 0.8860$)$, between 2,5 dimethylpyrazine and alanine-Ala $\left(R^{2} 0.8822\right)$, and pyrroles and the sum of ornithine-Orn and Alanine-Ala $\left(R^{2} 0.8604\right)$.

Results, although preliminary due to the limited sample set available, posed a solid foundation for the aroma potential concept; a comprehensive yet quantitative fingerprinting of hazelnut primary metabolome could be used as an effective tool to predict the aroma potential of hazelnuts (Cialiè Rosso et al., 2018). 


\section{CONCLUSIONS AND FUTURE PERSPECTIVES}

Modern investigation approaches inspired by "omics" strategies have the potentials to address most of the challenges posed by the study of complex food metabolome/volatilome in relation to biological phenomena (Miguel et al., 2011; Capozzi and Bordoni, 2013). In the case of hazelnuts, the effect of functional variables at the basis of phenotype expression, reaction to extreme climate events and local pedo-climatic conditions, changes during storage, odor quality, and hedonic profile have been observed, interpreted, and in several cases rationally modeled after effective and reliable exploration of the compositional complexity of entire volatilome.

Sensory guided strategies have identified key-aroma patterns evoking the unique and distinctive hazelnut flavor (Hofmann and Schieberle, 1995). However, it is still underexplored the role of ancillary odorants, i.e., those that do not exceed their odor perception threshold in the sample, that by interacting with ORs in the olfactory epithelium, might modulate the overall aroma perception with effects on hedonic properties (Cordero et al., 2019).

Moreover, efforts are needed to better understand the volatilome expression under the effect of key-functional variables, known to have a negative impact on sensory quality. Besides the autoxidation of lipids, responsible for the generation of potent odorants with unpleasant notes [e.g., $(E, E)-2,4$ nonadienal and $(E, E)$-2,4-decadienal - deep-fried; $(Z)$-2-octenal and (Z)-2-nonenal - fatty; hexanal - green; octanal - fatty, soapy], insights are expected on enzymatic degradation of lipids.

\section{REFERENCES}

Alamprese, C., Ratti, S., and Rossi, M. (2009). Effects of roasting conditions on hazelnut characteristics in a two-step process. J. Food Eng. 95, 272-279. doi: 10.1016/j.jfoodeng.2009.05.001

Alasalvar, C., Amaral, J. S., and Shahidi, F. (2006). Functional lipid characteristics of Turkish Tombul hazelnut (Corylus avellana L.). J. Agric. Food Chem. 54, 10177-10183. doi: 10.1021/jf061702w

Alasalvar, C., and Bolling, B. W. (2015). Review of nut phytochemicals, fat-soluble bioactives, antioxidant components and health effects. Br. J. Nutr. 113, S68-S78. doi: 10.1017/S0007114514003729

Alasalvar, C., Odabasi, A. Z., Demir, N., Balaban, M. Ö., Shahidi, F., and Cadwallader, K. R. (2004). Volatiles and flavor of five Turkish Hazelnut varieties as evaluated by descriptive sensory analysis, electronic nose, and dynamic headspace analysis/gas chromatography-mass spectrometry. J. Food Sci. doi: 10.1111/j.1365-2621.2004.tb13382.x

Alasalvar, C., Pelvan, E., and Amarowicz, R. (2010). Effects of roasting on tasteactive compounds of Turkish hazelnut varieties (Corylus avellana L.). J. Agric. Food Chem. 58, 8674-8679. doi: 10.1021/jf101039f

Alasalvar, C., Pelvan, E., Bahar, B., Korel, F., and Ölmez, H. (2012a). Flavour of natural and roasted Turkish hazelnut varieties (Corylus avellana L.) by descriptive sensory analysis, electronic nose and chemometrics. Int. J. Food Sci. Technol. 47, 122-131. doi: 10.1111/j.1365-2621.2011.02817.x

Alasalvar, C., and Shahidi, F. (2008). Tree Nuts: Composition, Phytochemicals, and Health Effects. doi: 10.1201/9781420019391.ch1

Alasalvar, C., Shahidi, F., and Cadwallader, K. R. (2003a). Comparison of natural and roasted Turkish Tombul hazelnut (Corylus avellana L.) volatiles and flavor
The evolution of free and esterified fatty acids along shelf life might have an impact on lipid oxidation stability. A better understanding of lipase/esterase activity could guide targeted actions to inhibit enzymes activity during post-harvest and storage (Cialiè Rosso et al., 2021).

The interconnection between primary and specialized nonvolatile metabolites patterns and volatiles generated during storage and industrial processing (i.e., dry-roasting), should be better explored. In this context, interesting outcomes could support the development of predictive models for odorant formation and aroma potential expression (Cialiè Rosso et al., $2018,2020)$ to be used as decision-makers at an industry level.

The industrial need for effective solutions to practical problems related to hazelnut quality management urges the adoption of multidisciplinary yet systemic approaches [i.e., omics strategies (Wishart, 2008; Ulaszewska et al., 2019)] giving access to a higher level of information closer to a better understanding of complex phenomena (Nanda and Das, 2011).

\section{AUTHOR CONTRIBUTIONS}

SS: data curation, validation, and writing-original draft, review and editing. FS, MC, and EL: writing review and editing. $\mathrm{CB}$ : funding acquisition, conceptualization, supervision, and writing review and editing. NS: project administration, conceptualization, and writing review and editing. GC and GG: conceptualization and writing review and editing. CC: funding acquisition, project administration, conceptualization, supervision, and writing-original draft, review and editing. All authors contributed to the article and approved the submitted version. by DHA/GC/MS and descriptive sensory analysis. J. Agric. Food Chem. 51, 5067-5072. doi: 10.1021/jf0300846

Alasalvar, C., Shahidi, F., Ohshima, T., Wanasundara, U., Yurttas, H. C., Liyanapathirana, C. M., et al. (2003b). Turkish Tombul hazelnut (Corylus avellana L.). 2. Lipid characteristics and oxidative stability. J. Agric. Food Chem. 51, 3797-3805. doi: 10.1021/jf021239x

Alasalvar, C., Topal, B., Serpen, A., Bahar, B., Pelvan, E., and Gökmen, V. (2012b). Flavor characteristics of seven grades of black tea produced in Turkey. J. Agric. Food Chem. 60, 6323-6332. doi: 10.1021/jf301498p

Amann, A., Costello, B., de, L., Miekisch, W., Schubert, J., Buszewski, B., et al. (2014). The human volatilome: volatile organic compounds (VOCs) in exhaled breath, skin emanations, urine, feces and saliva. J. Breath Res. 8, 34001. doi: 10.1088/1752-7155/8/3/034001

Amaral, J. S., Casal, S., Citov,á, I., Santos, A., Seabra, R. M., and Oliveira, B. P. P. (2006). Characterization of several hazelnut (Corylus avellana L.) cultivars based in chemical, fatty acid and sterol composition. Eur. Food Res. Technol. 222, 274-280. doi: 10.1007/s00217-005-0068-0

Amrein, T. M., Schwager, H., Meier, R., Frey, P., and Gassenmeier, K. (2014). A Metallic, Solvent-like Off-Flavor in Hazelnuts. Flavour Science (Elsevier Inc.) p. 661-664. doi: 10.1016/B978-0-12-398549-1.00121-5

Amrein, T. M., Schwager, H., Meier, R., Frey, P., and Gassenmeier, K. F. (2010). Identification of prenyl ethyl ether as a source of metallic, solvent-like off-flavor in Hazelnut. J. Agric. Food Chem. 58, 11408-412. doi: 10.1021/jf102334w

Artik, N., Akan, S., Okay, Y., Durmaz, N., and Köksal, A. I. (2021). Volatile aroma component of natural and roasted hazelnut varieties using solidphase microextraction gas chromatography/mass spectrometry. Acta Sci. Pol. Hortorum Cultus. 20, 85-96. doi: 10.24326/asphc.2021.5.8 
Audouze, K., Tromelin, A., Le Bon, A. M., Belloir, C., Petersen, R. K., Kristiansen, K., et al. (2014). Identification of odorant-receptor interactions by global mapping of the human odorome. PLoS ONE. 9. doi: 10.1371/journal.pone.0093037

Azarbad, M. H., and Jeleń, H. (2015). Determination of hexanal-an indicator of lipid oxidation by static headspace gas chromatography (SHS-GC) in fat-rich food matrices. Food Anal. Methods. doi: 10.1007/s12161-014-0043-0

Balik, H. I. I., Balik, S. K., Erdogan, V., Kafkas, S., Beyhan, N., Duyar, O., et al. (2018). Clonal selection in 'Tombul' hazelnut: preliminary results. Acta Hortic. 1226, 53-58. doi: 10.17660/ActaHortic.2018.1226.7

Belitz, H. D., Grosch, W., and Schieberle, P. (2009). Food Chemistry. 4th edition. Springer, Berlin, Heidelberg. doi: 10.1007/978-3-540-69934-7

Belviso, S., Dal Bello, B., Giacosa, S., Bertolino, M., Ghirardello, D., Giordano, M., et al. (2017). Chemical, mechanical and sensory monitoring of hot air- and infrared-roasted hazelnuts (Corylus avellana L.) during nine months of storage. Food Chem. 217, 398-408. doi: 10.1016/j.foodchem.2016.08.103

Bicchi, C., Cordero, C., and Rubiolo, P. (2004). A survey on high-concentrationcapability headspace sampling techniques in the analysis of flavors and fragrances. J. Chromatogr. Sci. 42, 402-409. doi: 10.1093/chromsci/42.8.402

Blanch, G. P., and Jauch, J. (1998). Enantiomeric composition of filbertone in hazelnuts in relation to extraction conditions. Multidimensional gas chromatography and gas chromatography/mass spectrometry in the single ion monitoring mode of a natural sample. J. Agric. Food Chem. 46, 4283-4286. doi: $10.1021 / \mathrm{j} 980272+$

Blank, I., Sen, A., and Grosch, W. (1992). Potent odorants of the roasted powder and brew of Arabica coffee. Z. Lebensm. Unters. Forsch. 195, 239-245. doi: $10.1007 / \mathrm{BF} 01202802$

Boccacci, P., and Botta, R. (2009). Investigating the origin of hazelnut (Corylus avellana L.) cultivars using chloroplast microsatellites. Genet. Resour. Crop Evol. 56, 851-859. doi: 10.1007/s10722-009-9406-6

Bonvehí, J. S., and Coll, F. V. (1993). Study of the carbohydrate fraction of the principal varieties of Tarragona hazelnuts (Corylus avellana L.). Food Chem. 46, 285-288. doi: 10.1016/0308-8146(93)90120-5

Bottone, A., Cerulli, A., Durso, G., Masullo, M., Montoro, P., Napolitano, A., et al. (2019). Plant specialized metabolites in hazelnut (Corylus avellana) kernel and byproducts: an update on chemistry, biological activity, and analytical aspects. Planta Med. doi: 10.1055/a-0947-5725

Bozoglu, M. (2005). The situation of the hazelnut sector in Turkey. Acta Hortic. 686, 641-648. doi: 10.17660/ActaHortic.2005.686.85

Breer, H., Fleischer, J., and Strotmann, J. (2006). The sense of smell: Multiple olfactory subsystems. Cell. Mol. Life Sci. 63, 1465-1475. doi: 10.1007/s00018-006-6108-5

Bressanello, D., Marengo, A., Cordero, C., Strocchi, G., Rubiolo, P., Pellegrino, G., et al. (2021). Chromatographic fingerprinting strategy to delineate chemical patterns correlated to coffee odor and taste attributes. J. Agric. Food Chem. doi: 10.1021/acs.jafc.1c00509

Broza, Y. Y., Mochalski, P., Ruzsanyi, V., Amann, A., and Haick, H. (2015). Hybrid volatolomics and disease detection. Angew. Chem. Int. Ed. Engl. 54, 11036-11048. doi: 10.1002/anie.201500153

Burdack-Freitag, A., and Schieberle, P. (2010). Changes in the key odorants of Italian hazelnuts (Coryllus avellana L. Var. Tonda Romana) induced by roasting. J. Agric. Food Chem. 58, 6351-6359. doi: 10.1021/jf100692k

Burdack-Freitag, A., and Schieberle, P. (2012). Characterization of the key odorants in raw italian hazelnuts (Corylus avellana L. var. Tonda Romana) and roasted hazelnut paste by means of molecular sensory science. J. Agric. Food Chem. 60, 5057-5064. doi: 10.1021/jf300908d

Caligiani, A., Coisson, J. D., Travaglia, F., Acquotti, D., Palla, G., Palla, L., et al. (2014). Application of $1 \mathrm{H}$ NMR for the characterisation and authentication of "Tonda Gentile Trilobata" hazelnuts from Piedmont (Italy). Food Chem. 148, 77-85. doi: 10.1016/j.foodchem.2013.10.001

Capozzi, F., and Bordoni, A. (2013). Foodomics: a new comprehensive approach to food and nutrition. Genes Nutr. 8, 1-4. doi: 10.1007/s12263-012-0310-x

Caramiello, R., Me, G., and Radicati, L. (2000). Structure and characteristics of the hazelnut shell in different cultivars and their agronomic and industrial influence. Acta Hortic., 195-208. doi: 10.17660/ActaHortic.2000.517.23

Charve, J., Chen, C., Hegeman, A. D., and Reineccius, G. A. (2011). Evaluation of instrumental methods for the untargeted analysis of chemical stimuli of orange juice flavour. Flavour Fragr. J. 26, 429-440. doi: 10.1002/ffj.2078
Chetschik, I., Granvogl, M., and Schieberle, P. (2010). Quantitation of key peanut aroma compounds in raw peanuts and pan-roasted peanut meal, aroma reconstitution and comparison with commercial peanut products. J. Agric. Food Chem. 58, 11018-11026. doi: 10.1021/jf1026636

Cialiè Rosso, M. (2020). Advanced analytical approaches for "omic" investigations of high quality food matrices of vegetable origin (Ph.D. thesis). University of Turi, Turin, Italy. Available online at: https://dott-sfb.campusnet.unito.it/att/XXXII/ Abstract_CIALIE_ROSSO.pdf

Cialiè Rosso, M., Liberto, E., Spigolon, N., Fontana, M., Somenzi, M., Bicchi, C., et al. (2018). Evolution of potent odorants within the volatile metabolome of high-quality hazelnuts (Corylus avellana L.): evaluation by comprehensive two-dimensional gas chromatography coupled with mass spectrometry. Anal. Bioanal. Chem. 410, 3491-3506. doi: 10.1007/s00216-017-0832-6

Cialiè Rosso, M., Mazzucotelli, M., Bicchi, C., Charron, M., Manini, F., Menta, R., et al. (2020). Adding extra-dimensions to hazelnuts primary metabolome fingerprinting by comprehensive two-dimensional gas chromatography combined with time-of-flight mass spectrometry featuring tandem ionization: Insights on the aroma potential. J. Chromatogr. A. 1614, 1-11. doi: 10.1016/j.chroma.2019.460739

Cialiè Rosso, M., Stilo, F., Mascrez, S., Bicchi, C., Purcaro, G., and Cordero, C. (2021). Shelf-life evolution of the fatty acid fingerprint in high-quality hazelnuts (Corylus avellana 1.) harvested in different geographical regions. Foods. doi: 10.3390/foods10030685

Ciarmiello, L. F., Mazzeo, M. F., Minasi, P., Peluso, A., De Luca, A., Piccirillo, P., et al. (2014). Analysis of different European hazelnut (corylus avellana L.) cultivars: Authentication, phenotypic features, and phenolic profiles. J. Agric. Food Chem. 62, 6236-6246. doi: 10.1021/jf5018324

Ciarmiello, L. F., Piccirillo, P., Gerardi, C., Piro, F., Luca, A., De, D’Imperio, F., et al. (2013). Microwave irradiation for dry-roasting of hazelnuts and evaluation of microwave treatment on hazelnuts peeling and fatty acid oxidation. J. Food Res. 2, 22. doi: 10.5539/jfr.v2n3p22

Coelho, C. M. M., Bellato, C., de, M., Santos, J. C. P., Ortega, E. M. M., and Tsai, S. M. (2007). Effect of phytate and storage conditions on the development of the 'hard-to-cook.' J. Sci. Food Agric. 1243, 1237-1243. doi: 10.1002/jsfa.2822

Cordero, C., Bicchi, C., and Rubiolo, P. (2008). Group-type and fingerprint analysis of roasted food matrices (coffee and hazelnut samples) by comprehensive two-dimensional gas chromatography. J. Agric. Food Chem. 56, 7655-7666. doi: $10.1021 / \mathrm{jf} 801001 \mathrm{z}$

Cordero, C., Kiefl, J., Reichenbach, S. E., and Bicchi, C. (2019). Characterization of odorant patterns by comprehensive two-dimensional gas chromatography: a challenge in omic studies. TrAC - Trends Anal. Chem. 113, 364-378. doi: $10.1016 / j . t r a c .2018 .06 .005$

Cordero, C., Kiefl, J., Schieberle, P., Reichenbach, S. E., and Bicchi, C. (2015). Comprehensive two-dimensional gas chromatography and food sensory properties: potential and challenges. Anal. Bioanal. Chem. 407, 169-191. doi: 10.1007/s00216-014-8248-z

Cordero, C., Liberto, E., Bicchi, C., Rubiolo, P., Schieberle, P., Reichenbach, S. E., et al. (2010). Profiling food volatiles by comprehensive two-dimensional ga schromatography coupled with mass spectrometry: Advanced fingerprinting approaches for comparative analysis of the volatile fraction of roasted hazelnuts (Corylus avellana L.) from different ori. J. Chromatogr. A. 1217, 5848-5858. doi: 10.1016/j.chroma.2010.07.006

Cristofori, V., Ferramondo, S., Bertazza, G., and Bignami, C. (2008). Nut and kernel traits and chemical composition of hazelnut (Corylus avellana L.) cultivars. J. Sci. Food Agric. 88, 1091-1098. 3203. doi: 10.1002/jsf a. 3203

Dairou, V., and Sieffermann, J. M. (2002). A comparison of 14 jams characterized by conventional profile and a quick original method, the Flash Profile. J. Food Sci. 67, 826-834. doi: 10.1111/j.1365-2621.2002.tb10685.x

Delarue, J., and Sieffermann, J. M. (2004). Sensory mapping using Flash profile. Comparison with a conventional descriptive method for the evaluation of the flavour of fruit dairy products. Food Qual. Prefer. 15, 383-392. doi: 10.1016/S0950-3293(03)00085-5

Dewick, P. M. (1986). The Biochemistry of Plant Phenolics. Phytochemistry. 25, 2011-2012. doi: 10.1016/S0031-9422(00)81203-0

Donno, D., Beccaro, G. L., Mellano, G. M., Di Prima, S., Cavicchioli, M., Cerutti, A. K., et al. (2013). Setting a protocol for hazelnut roasting using sensory and colorimetric analysis: Influence of the roasting temperature on the quality 
of tonda gentile delle langhe cv. Hazelnut. Czech J. Food Sci. 31, 390-400. doi: 10.17221/390/2012-CJFS

Dunkel, A., Steinhaus, M., Kotthoff, M., Nowak, B., Krautwurst, D., Schieberle, P., et al. (2014). Nature's chemical signatures in human olfaction: A foodborne perspective for future biotechnology. Angew. Chemie - Int. Ed. 53, 7124-7143. doi: 10.1002/anie. 201309508

Durak, I., Koksal, I., Kacmaz, M., Buyukkocak, S., Cimen, B. M. Y., and Ozturk, H. S. (1999). Hazelnut supplementation enhances plasma antioxidant potential and lowers plasma cholesterol levels. Clin. Chim. Acta. 284, 113-115. doi: 10.1016/S0009-8981(99)00066-2

Erdogan, V., and Mehlenbacher, S. A. (2000). Interspecific hybridization in hazelnut (Corylus). J. Am. Soc. Hortic. Sci. 125, 489-497. doi: 10.21273/JASHS.125.4.489

Fanali, C., Tripodo, G., Russo, M., Della Posta, S., Pasqualetti, V., and De Gara, L. (2018). Effect of solvent on the extraction of phenolic compounds and antioxidant capacity of hazelnut kernel. Electrophoresis. doi: 10.1002/elps.201800014

FAO (2019). FAOSTAT - Production quantities of hazelnuts (2013-2017).

Firestein, S. (2001). How the olfactory system makes sense of scent. Nature. 413, 211-218. doi: 10.1038/35093026

Franchina, F. A., Maimone, M., Tranchida, P. Q., and Mondello, L. (2016). Flow modulation comprehensive two-dimensional gas chromatography-mass spectrometry using $\approx 4 \mathrm{~mL} \mathrm{~min}-1$ gas flows. J. Chromatogr. A. 1441, 134-139. doi: 10.1016/j.chroma.2016.02.041

Frauendorfer, F., and Schieberle, P. (2008). Changes in key aroma compounds of Criollo cocoa beans during roasting. J. Agric. Food Chem. 56, 10244-10251. doi: $10.1021 /$ jf802098f

Frega, N., Mozzon, M., and Lercker, G. (1999). Effects of free fatty acids on oxidative stability of vegetable oil. JAOCS J. Am. Oil Chem. Soc. 76, 325-329. doi: 10.1007/s11746-999-0239-4

Fridman, E. (2005). Metabolic, genomic, and biochemical analyses of glandular trichomes from the wild tomato species lycopersicon hirsutum identify a key enzyme in the biosynthesis of methylketones. Plant Cell Online. 17, 1252-1267. doi: $10.1105 /$ tpc. 104.029736

Ghirardello, D., Bertolino, M., Belviso, S., Dal Bello, B., Giordano, M., Rolle, L., et al. (2016). Phenolic composition, antioxidant capacity and hexanal content of hazelnuts (Corylus avellana L.) as affected by different storage conditions. Postharvest Biol. Technol. 112, 95-104. doi: 10.1016/j.postharvbio.2015.09.039

Ghirardello, D., Contessa, C., Valentini, N., Zeppa, G., Rolle, L., Gerbi, V., et al. (2013). Effect of storage conditions on chemical and physical characteristics of hazelnut (Corylus avellana L.). Postharvest Biol. Technol. 81, 37-43. doi: 10.1016/j.postharvbio.2013.02.014

Ghisoni, S., Lucini, L., Rocchetti, G., Chiodelli, G., Farinelli, D., Tombesi, S., et al. (2020). Untargeted metabolomics with multivariate analysis to discriminate hazelnut (Corylus avellana L.) cultivars and their geographical origin. J. Sci. Food Agric. 100, 500-508. doi: 10.1002/jsfa.9998

Giddings, J. C. (1995). Sample dimensionality: A predictor of order-disorder in component peak distribution in multidimensional separation. J. Chromatogr. A. 703, 3-15. doi: 10.1016/0021-9673(95)00249-M

Giraudo, A., Calvini, R., Orlandi, G., Ulrici, A., Geobaldo, F., and Savorani, F. (2018). Development of an automated method for the identification of defective hazelnuts based on RGB image analysis and colourgrams. Food Control. 94, 233-240. doi: 10.1016/j.foodcont.2018.07.018

Goff, S. A., and Klee, H. J. (2006). Plant volatile compounds : sensory. Science. 311, 815-819. doi: 10.1126/science. 1112614

Göncüoglu Taş, N., and Gökmen, V. (2017). Maillard reaction and caramelization during hazelnut roasting: a multiresponse kinetic study. Food Chem. 221, 1911-1922. doi: 10.1016/j.foodchem.2016.11.159

Grosch, W. (2001). Evaluation of the key odorants of foods by dilution experiments, aroma models and omission. Chem. Sens. 26, 533-545. doi: $10.1093 /$ chemse/26.5.533

Güntert, M., Emberger, R., Hopp, R., Köpsel, M., Silberzahn, W., and Werkhoff, P. (1991). Chirospecific analysis in flavor and essential oil chemistry Part A. Filbertone - the character impact compound of hazel-nuts. Z. Lebensm. Unters. Forsch. 192, 108-110. doi: 10.1007/BF01202621

Han, B. Z., Rombouts, F. M., and Nout, M. J. R. (2001). A Chinese fermented soybean food. Int. J. Food Microbiol. 65, 1-10. doi: 10.1016/S0168-1605(00)00523-7
Hofmann, T., and Schieberle, P. (1995). Evaluation of the key odorants in a thermally treated solution of ribose and cysteine by aroma extract dilution techniques. J. Agric. Food Chem. 43, 2187-2194. doi: 10.1021/jf00056a042

Hofmann, T., and Schieberle, P. (1998). Flavor contribution and formation of the intense roast-smelling odorants 2-propionyl-1-pyrroline and 2propionyltetrahydropyridine in maillard-type reactions. J. Agric. Food Chem. 46, 2721-2726. doi: 10.1021/jf971101s

Hung, R., Lee, S., and Bennett, J. W. (2014). The effects of low concentrations of the enantiomers of mushroom alcohol (1-octen-3-ol) on Arabidopsis thaliana. Mycology. 5, 73-80. doi: 10.1080/21501203.2014.902401

Ilyasoglu, H. (2015). Changes in sterol composition of hazelnut during fruit development. Int. J. Food Prop. 18, 456-463. doi: 10.1080/10942912.2013.837065

ISLAM, A. (2018). Hazelnut cultivation in Turkey. Akad. Ziraat Derg. 7, 251-258. doi: $10.29278 /$ azd. 476665

Jauch, J., Schmalzing, D., Schurig, V., Emberger, R., Hopp, R., Köpsel, M., et al. (1989). Isolation, Synthesis, and Absolute Configuration of Filbertone - the Principal Flavor Component of the Hazelnut. Angew. Chemie Int. Ed. English 28, 1022-1023. doi: 10.1002/anie.198910221

Kamal-Eldin, A., and Moreau, R. A. (2009). Tree nut oils. In: Gourmet and Health-Promoting Specialty Oils (AOCS Press). p. 127-149. doi: 10.1016/B978-1-893997-97-4.50009-7

Kiefl, J. (2013). Differentiation of Hazelnut Cultivars (Corylus avellana L.) by Metabolomics and Sensomics Approaches Using Comprehensive Twodimensional Gas Chromatography Time-Of-Flight Mass Spectrometry $(G C \times G C / T O F-M S)$. doi: 10.1021/jf400807w

Kiefl, J., Cordero, C., Nicolotti, L., Schieberle, P., Reichenbach, S. E., and Bicchi, C. (2012). Performance evaluation of non-targeted peak-based crosssample analysis for comprehensive two-dimensional gas chromatographymass spectrometry data and application to processed hazelnut profiling. J. Chromatogr. A 1243, 81-90. doi: 10.1016/j.chroma.2012.04.048

Kiefl, J., Pollner, G., and Schieberle, P. (2013). Sensomics analysis of key hazelnut odorants (Corylus avellana L.'Tonda Gentile') using comprehensive two-dimensional gas chromatography in combination with time-of-flight mass spectrometry (GC $\times$ GC-TOF-MS). J. Agric. Food Chem. 61, 5226-5235. doi: $10.1021 /$ jf400807

Kiefl, J., and Schieberle, P. (2013). Evaluation of process parameters governing the aroma generation in three hazelnut cultivars (Corylus avellana L.) by correlating quantitative key odorant profiling with sensory evaluation. J. Agric. Food Chem. 61, 5236-5244. doi: 10.1021/jf4008086

Kinderlerer, J. L., and Johnson, S. (1992). Rancidity in hazelnuts due to volatile aliphatic aldehydes. J. Sci. Food Agric. 58, 89-93. doi: 10.1002/jsfa.27405 80115

Köksal, A. I., Artik, N., Simşek, A., and Güneş, N. (2006). Nutrient composition of hazelnut (Corylus avellana L.) varieties cultivated in Turkey. Food Chem. 99, 509-515. doi: 10.1016/j.foodchem.2005.08.013

Koyuncu, M. A., Islam, A., and Küçük, M. (2005). Fat and fatty acid composition of hazelnut kernels in vacuum packages during storage. Grasas y Aceites 56, 263-266. doi: 10.3989/gya.2005.v56.i4.91

Locatelli, M., Coïsson, J. D., Travaglia, F., Bordiga, M., and Arlorio, M. (2015). Impact of roasting on identification of hazelnut (Corylus avellana L.) origin: a chemometric approach. J. Agric. Food Chem. 63, 7294-7303. doi: 10.1021/acs.jafc.5b03201

Magagna, F., Liberto, E., Reichenbach, S. E., Tao, Q., Carretta, A., Cobelli, L., et al. (2018). Advanced fingerprinting of high-quality cocoa: Challenges in transferring methods from thermal to differential-flow modulated comprehensive two dimensional gas chromatography. J. Chromatogr. A. 1536, 122-136. doi: 10.1016/j.chroma.2017.07.014

Meadow, J. F., Altrichter, A. E., Bateman, A. C., Stenson, J., Brown, G. Z., Green, J. L., et al. (2015). Humans differ in their personal microbial cloud. Peer J. 3, e1258. doi: $10.7717 /$ peerj. 1258

Mehlenbacher, S. A., Smith, D. C., and Brenner, L. K. (1993). Variance components and heritability of nut and kernel defects in hazelnut. Plant Breed. 110, 144-152. doi: 10.1111/j.1439-0523.1993.tb01226.x

Memoli, A., Albanese, D., Esti, M., Lombardelli, C., Crescitelli, A., Di Matteo M., et al. (2017). Effect of bug damage and mold contamination on fatty acids and sterols of hazelnut oil. Eur. Food Res. Technol. 243, 651-658. doi: $10.1007 / \mathrm{s} 00217-016-2778-\mathrm{x}$ 
Miguel, H., Carolina, S., Virginia, G., Elena, I., and Alejandro, C. (2011). Foodomics: MS-based strategies in modern food science and nutrition. Mass Spectrom. Rev. 31, 49-69. doi: 10.1002/mas.20335

Momchilova, S. M., Taneva, S. P., Zlatanov, D., Antova, G. A., Angelova-Romova, M. J., and Blagoeva, E. (2017). Fatty acids, tocopherols and oxidative stability of hazelnuts during storage. Bulg. Chem. Commun. 49, 65-70.

Nanda, T., and Das, M. (2011). Metabolomics: the future of systems biology. J. Comput. Sci. Syst. Biol. 4. doi: 10.4172/jcsb.S13-003

Nicolotti, L., Cordero, C., Bicchi, C., Rubiolo, P., Sgorbini, B., and Liberto, E. (2013a). Volatile profiling of high quality hazelnuts (Corylus avellana L.): Chemical indices of roasting. Food Chem. 138, 1723-1733. doi: 10.1016/j.foodchem.2012.11.086

Nicolotti, L., Cordero, C., Cagliero, C., Liberto, E., Sgorbini, B., Rubiolo, P., et al. (2013b). Quantitative fingerprinting by headspace-Two-dimensional comprehensive gas chromatography-mass spectrometry of solid matrices: some challenging aspects of the exhaustive assessment of food volatiles. Anal. Chim. Acta 798, 115-125. ,0.2013.08.052. doi: 10.1016/j.aca.2013.08.052

ÖZDEMIR, M. (1998). Factors influencing shelf life of hazelnut. Basimi-Published Gida Teknol. 3, 66-71.

Özdemir, M., Açkurt, F., Yildiz, M., Biringen, G., Gürcan, T., and Löker, M. (2001). Effect of roasting on some nutrients of hazelnuts (Corylus Avellena L.). Food Chem. 73, 185-190. doi: 10.1016/S0308-8146(00)00260-0

Parcerisa, J., Codony, R., Boatella, J., and Rafecas, M. (1999). Fatty acids including trans content of commercial bakery products manufactured in Spain. J. Agric. Food Chem. 47, 2040-2043. doi: 10.1021/jf980941j

Pastorelli, S., Torri, L., Rodriguez, A., Valzacchi, S., Limbo, S., and Simoneau, C. (2007). Solid-phase micro-extraction (SPME-GC) and sensors as rapid methods for monitoring lipid oxidation in nuts. Food Addit. Contam. 24, 1219-1225. doi: 10.1080/02652030701426987

Pedrotti, M., Khomenko, I., Genova, G., Castello, G., Spigolon, N., Fogliano, V., et al. (2021). Quality control of raw hazelnuts by rapid and noninvasive fingerprinting of volatile compound release. LWT 143, 111089. doi: 10.1016/j.lwt.2021.111089

Phillips, M., Cataneo, R. N., Chaturvedi, A., Kaplan, P. D., Libardoni, M., Mundada, M., et al. (2013). Detection of an extended human volatome with comprehensive two-dimensional gas chromatography time-of-flight mass spectrometry. PLoS ONE. 8, e75274. doi: 10.1371/journal.pone.0075274

Prosperini, S., Ghirardello, D., Scursatone, B., Gerbi, V., and Zeppa, G. (2009). Identification of soluble phenolic acids in hazelnut (Corylus avellana L.) kernel. In: Seventh International Congress on Hazelnut. vol. 845, pp. 677-680. doi: 10.17660/ActaHortic.2009.845.106

Pscheidt, J. W., Heckert, S., Wiseman, M., and Jones, L. (2019). Fungi associated with and influence of moisture on development of kernel mold of hazelnut. Plant Dis. 103, 922-928. doi: 10.1094/PDIS-09-18-1520-RE

Puchl'ová, E., and Szolcsányi, P. (2018). Filbertone: a review. J. Agric. Food Chem. 66, 11221-11226. doi: 10.1021/acs.jafc.8b04332

Ramalhosa, E., Delgado, T., Estevinho, L., and Pereira, J. A. (2011). Hazelnut (Corylus avellana L.) Cultivars and Antimicrobial Activity. In: Nuts and Seeds in Health and Disease Prevention, p. 627-636. doi: 10.1016/B978-0-12-375688-6.10073-8

Romero-Guido, C., Belo, I., Ta, T. M. N., Cao-Hoang, L., Alchihab, M., Gomes, N., et al. (2011). Biochemistry of lactone formation in yeast and fungi and its utilisation for the production of flavour and fragrance compounds. Appl. Microbiol. Biotechnol. 89, 535-547. doi: 10.1007/s00253-010-2945-0

Rosso, M. C., Stilo, F., Bicchi, C., Charron, M., Rosso, G., Menta, R., et al. (2021). Combined untargeted and targeted fingerprinting by comprehensive two-dimensional gas chromatography to track compositional changes on hazelnut primary metabolome during roasting. Appl. Sci. 11, 1-18. doi: 10.3390/app11020525

Ruiz del Castillo, M. L., Flores, G., Herraiz, M., and Blanch, G. P. (2003). Solid-phase microextraction for studies on the enantiomeric composition of filbertone in hazelnut oils. J. Agric. Food Chem. 51, 2496-2500. doi: $10.1021 /$ jf026247s

Saklar, S., Katnas, S., and Ungan, S. (2001). Determination of optimum hazelnut roasting conditions. Int. J. Food Sci. Technol. 36, 271-281. doi: 10.1046/j.1365-2621.2001.00457.x

Saklar, S., Ungan, S., and Katnas, S. (2003). Microstructural changes in hazelnuts during roasting. Food Res. Int. 36, 19-23. doi: 10.1016/S0963-9969(02)00103-5
Schieberle, P., and Hofmann, T. (2011). Mapping the combinatorial code of food flavors by means of molecular sensory science approach. In: Food Flavors: Chemical, Sensory and Technological Properties. p. 413-438.

Schnermann, P., and Schieberle, P. (1997). Evaluation of key odorants in milk chocolate and cocoa mass by aroma extract dilution analyses. J. Agric. Food Chem. 45, 867-872. doi: 10.1021/jf960670h

Sciubba, F., Di Cocco, M. E., Gianferri, R., Impellizzeri, D., Mannina, L., De Salvador, F. R., et al. (2014). Metabolic profile of different Italian cultivars of hazelnut (Corylus avellana) by nuclear magnetic resonance spectroscopy. Nat. Prod. Res. 28, 1075-1081. doi: 10.1080/14786419.2014.905936

Seyhan, F., Ozay, G., Saklar, S., Erta,ş, E., Satir, G., and Alasalvar, C. (2007). Chemical changes of three native Turkish hazelnut varieties (Corylus avellana L.) during fruit development. Food Chem. 105, 590-596. doi: 10.1016/j.foodchem.2007.04.016

Sgorbini, B., Cagliero, C., Liberto, E., Rubiolo, P., Bicchi, C., and Cordero, C. E. I. (2019). Strategies for accurate quantitation of volatiles from foods and plant-origin materials: a challenging task. J. Agric. Food Chem. 67, 1619-1630. doi: 10.1021/acs.jafc.8b06601

Shahidi, F., Alasalvar, C., and Liyana-Pathirana, C. M. (2007). Antioxidant phytochemicals in hazelnut kernel (Corylus avellana L) and hazelnut byproducts. J. Agric. Food Chem. 55, 1212-1220. doi: 10.1021/jf062472o

Sides, A., Robards, K., and Helliwell, S. (2000). Developments in extraction techniques and their application to analysis of volatiles in foods. $\operatorname{Tr} A C$ - Trends Anal. Chem. 19, 322-329. doi: 10.1016/S0165-9936(99)00225-3

Singldinger, B., Dunkel, A., Bahmann, D., Bahmann, C., Kadow, D., Bisping, B., et al. (2018). New Taste-Active 3-(O- $\beta$ - $d$-Glucosyl)-2-oxoindole-3-acetic acids and diarylheptanoids in cimiciato-infected hazelnuts. J. Agric. Food Chem. 66, 4660-4673. doi: 10.1021/acs.jafc.8b01216

Stilo, F., Bicchi, C., Reichenbach, S. E., and Cordero, C. (2021a). Comprehensive two-dimensional gas chromatography as a boosting technology in food-omic investigations. J. Sep. Sci. 44, 1592-1611. doi: 10.1002/jssc.202100017

Stilo, F., Liberto, E., Spigolon, N., Genova, G., Rosso, G., Fontana, M., et al. (2021b). An effective chromatographic fingerprinting workflow based on comprehensive two-dimensional gas chromatography - Mass spectrometry to establish volatiles patterns discriminative of spoiled hazelnuts (Corylus avellana L.). Food Chem. 340, 128135. doi: 10.1016/j.foodchem.2020.128135

Taş, N. G., and Gökmen, V. (2018). Profiling of the Contents of Amino Acids, Water-Soluble Vitamins, Minerals, Sugars and Organic Acids in Turkish Hazelnut Varieties. Polish J. Food Nutr. Sci. 68, 223-234. doi: 10.1515/pjfns-2018-0002

Tranchida, P. Q., Donato, P., Cacciola, F., Beccaria, M., Dugo, P., and Mondello, L. (2013). Potential of comprehensive chromatography in food analysis. $\operatorname{Tr} A C$ - Trends Anal. Chem. 52, 186-205. doi: 10.1016/j.trac.2013.07.008

Turan, A. (2018). Effect of drying methods on fatty acid profile and oil oxidation of hazelnut oil during storage. Eur. Food Res. Technol. 244, 2181-2190. doi: 10.1007/s00217-018-3128-y

Ulaszewska, M. M., Weinert, C. H., Trimigno, A., Portmann, R., Andres Lacueva C., Badertscher, R., et al. (2019). Nutrimetabolomics: an integrative action for metabolomic analyses in human nutritional studies. Mol. Nutr. Food Res. 63, 1-38. doi: 10.1002/mnfr.201800384

Van Boekel, M. A. J. S. (2006). Formation of flavour compounds in the Maillard reaction. Biotechnol. Adv. 24, 230-233. doi: 10.1016/j.biotechadv.2005. 11.004

Van Gemert, L. J. (2003). Odour Thresholds. Compil. odour Threshold values air, water other media.

Weber, B., and Mosandl, A. (1997). Stereoisomeric flavour compounds LXXV: Synthesis and structure-function relationship of 3methylthiobutanal enantiomers. Eur. Food Res. Technol. 204, 194-197. doi: $10.1007 / \mathrm{s} 002170050061$

Wishart, D. S. (2008). Metabolomics: applications to food science and nutrition research. Trends Food Sci. Technol. 19, 482-493. doi: 10.1016/j.tifs.2008. 03.003

Yuan, B., Lu, M., Eskridge, K. M., Isom, L. D., and Hanna, M. A. (2018). Extraction, identification, and quantification of antioxidant phenolics from hazelnut (Corylus avellana L.) shells. Food Chem. 244, 7-15. doi: 10.1016/j.foodchem.2017.09.116

Zabaras, D., and Gordon, M. H. (2004). Detection of pressed hazelnut oil in virgin olive oil by analysis of polar components: Improvement and validation 
of the method. Food Chem. 84, 475-483. doi: 10.1016/j.foodchem.2003. 07.029

Zawirska-wojtasiak, R. (2006). Chirality and the nature of food authenticity of aroma. Acta Sci. Pol. Technol. Aliment. 5, 21-36. Available online at: https:// www.food.actapol.net/pub/2_1_2006.pdf

Zhou, K., Slavin, M., Lutterodt, H., Whent, M., and Yu, L. (2013). Biochemistry of Foods.

Conflict of Interest: FS was employed by Laemmegroup, a Tentamus Company. NS, GC, and GG are employees of Soremartec Italia srl, a Ferrero Group company.

The remaining authors declare that the research was conducted in the absence of any commercial or financial relationships that could be construed as a potential conflictof interest.
Publisher's Note: All claims expressed in this article are solely those of the authors and do not necessarily represent those of their affiliated organizations, or those of the publisher, the editors and the reviewers. Any product that may be evaluated in this article, or claim that may be made by its manufacturer, is not guaranteed or endorsed by the publisher.

Copyright (C) 2022 Squara, Stilo, Cialiè Rosso, Liberto, Spigolon, Genova, Castello, Bicchi and Cordero. This is an open-access article distributed under the terms of the Creative Commons Attribution License (CC BY). The use, distribution or reproduction in other forums is permitted, provided the original author(s) and the copyright owner(s) are credited and that the original publication in this journal is cited, in accordance with accepted academic practice. No use, distribution or reproduction is permitted which does not comply with these terms. 